\title{
FluorMODleaf: A new leaf fluorescence emission model based on the PROSPECT model
}

\author{
R. Pedrós ${ }^{\mathrm{a}, \mathrm{b}, \mathrm{c}}$, Y. Goulas ${ }^{\mathrm{b}, *}, \mathrm{~S}$. Jacquemoud ${ }^{\mathrm{c}}$, J. Louis ${ }^{\mathrm{d}}$, I. Moya ${ }^{\mathrm{b}}$ \\ a Solar Radiation Group, Department of Earth Physics and Thermodynamics, University of Valencia, Spain \\ b Equipe de Fluorescence, Laboratoire de Météorologie Dynamique, Centre National de la Recherche Scientifique, UMR 8359, Palaiseau, France \\ c Equipe de Géophysique Spatiale et Planétaire, Institut de Physique du Globe de Paris et Université Paris Diderot, UMR 7154, Paris, France \\ d Equipe de Biospectroscopie Végétale, Laboratoire Ecologie Systématique et Evolution, UMR 8079, Orsay, France
}

\section{A R T I C L E I N F O}

\section{Article history:}

Received 4 March 2009

Received in revised form 26 August 2009

Accepted 29 August 2009

\section{Keywords:}

Solar-induced chlorophyll fluorescence

Reflectance

Transmittance

Leaf optical properties

Vegetation remote sensing

Radiative transfer model

\begin{abstract}
A B S T R A C T
A new model of chlorophyll $a$ fluorescence emission by plant leaves, FluorMODleaf, is presented. It is an extension of PROSPECT, a widely used leaf optical properties model that regards the leaf as a pile of $N$ absorbing and diffusing elementary plates. In FluorMODleaf, fluorescence emission of an infinitesimal layer of thickness $d x$ is integrated over the entire elementary plate. The fluorescence source function is based on the excitation spectrum of diluted isolated thylakoids and on the emission spectra of isolated photosystems, PSI and PSII, which are the main pigment-protein complexes involved in the initial stages of photosynthesis. Scattering within the leaf is produced by multiple reflections within and between elementary plates. The input variables of FluorMODleaf are: the number of elementary plates $N$, also called leaf structure parameter, the total chlorophyll content $C_{a b}$, the total carotenoid content $C_{C X}$, the equivalent water thickness $C_{w}$, and the dry matter content $C_{m}$ (or leaf mass per area), as in the new PROSPECT-5, plus the $\sigma_{I I} / \sigma_{I}$ ratio referring to the relative absorption cross section of PSI and PSII, and the fluorescence quantum efficiency of PSI and PSII, $\tau_{I}$ and $\tau_{I I}$, that are introduced here as mean fluorescence lifetimes. The model, which considers the reabsorption of emitted light within the leaf, allows good quantitative estimation of both upward and downward apparent spectral fluorescence yield (ASFY) at different excitation wavelengths from $400 \mathrm{~nm}$ to $700 \mathrm{~nm}$. It also emphasizes the role of scattering in fluorescence emission by leaves having high chlorophyll content.
\end{abstract}

(c) 2009 Elsevier Inc. All rights reserved.

\section{Introduction}

In the past 30 years, Earth observation from space has demonstrated its ability to monitor changes at a global scale. The complexity of physical, chemical, and biological processes that occur in terrestrial ecosystems requires detailed characterization of the natural environment and assessment of the interactions between soil, vegetation, and atmosphere to understand biosphere functioning (Monteith \& Unsworth, 2007). This can be fulfilled by coupling physics-based canopy functioning models with remote sensing data that also require advanced quantitative methods to exploit information of all kinds present in the radiometric signal. Since they are not directly linked to fundamental processes of plant physiology, like photosynthesis, multispectral and/or multiangular reflectance factors measured by present optical sensors only provide partial information on vegetation dynamics, especially on a short timescale. In other words, one cannot monitor photosynthesis using only vegetation reflectance. Chlorophyll fluorescence $(\mathrm{ChlF})$ is known to carry very specific information

* Corresponding author. Laboratoire de Météorologie Dynamique, Ecole Polytechnique, F91128 Palaiseau Cedex, France.

E-mail address: yves.goulas@lmd.polytechnique.fr (Y. Goulas). about leaf light-use efficiency, i.e., plant vitality and biomass productivity. Passive remote sensing techniques like the measurement of solar-induced chlorophyll fluorescence, which is modulated by photosynthetic efficiency, should improve our knowledge of the terrestrial carbon cycle and help us monitoring vegetation health from airborne or spaceborne platforms (Moya et al. 1992; Davidson et al., 2003; Moya \& Cerovic, 2004; Grace et al., 2007; Guanter et al., 2007).

Both at leaf and canopy levels, fluorescence emission is an intricate response of environmental and physiological factors. To sort them out and better interpret this signal, the European Space Agency funded the FluorMOD project (ESA contract no. 16365/02/NL/FF) that aimed at developing an integrated top-of-atmosphere vegetation fluorescence model (Miller et al., 2005). A detailed description of light interaction with single vegetation elements, i.e., plant leaves, both at excitation and emission wavelengths, is the first link in the chain that will permit full exploitation of the fluorescence emission signal measured by a remote sensor.

Most fluorescence models have been developed for other applications than plant physiology, like medical diagnosis of human diseases (e.g., Wu et al., 1993; Georgakoudi, 2006; Gupta et al., 2006) or quality color control for the paper industry (e.g., Emmel, 1998, 2000; Emmel \& Hersch, 1998) where fluorescence spectroscopy is widely used. In 
most media, the measured emission spectrum is distorted due to light reabsorption and/or scattering, which makes its interpretation especially difficult. Different theories have emerged that attempt to explain the propagation of light in a turbid medium, including fluorescence emission: radiative transfer equation (Richards-Kortum et al., 1989; Gardner et al., 1996; Emmel \& Hersch, 1998), two-flux Kubelka-Munk (KM) theory and four-flux extensions (Allen, 1964; Fukshansky \& Kazarinova, 1980; Bonham, 1986; Shakespeare \& Shakespeare, 2003), Monte Carlo simulations (Wu et al., 1993; Crilly et al., 1997; Welch et al., 1997), or rigorous analysis of tissue fluorescence based on electromagnetic theory (Panou-Diamandi et al., 1998).

Conversely, there are few leaf fluorescence models in the literature. They are based on different theories of light propagation within a plant leaf. The simplest ones assume an exponential light decay within the blade, which follows Beer's law (Agati et al., 1993; Ounis et al., 2001). The Kubelka-Munk differential equations are preferred by Rosema et al. (1991), Zarco-Tejada et al. (2000), and Ramos and Lagorio (2004) who solve the system analytically by successive approximations or numerically using the adding-doubling technique. Other approaches like Markov chains (Maier, 2000) or Monte Carlo photon transport (Sušila \& Nauš, 2007) have also been developed. The main challenge in leaf fluorescence modeling lies in the accurate simulation of light reabsorption and its impact on fluorescence emission. For this purpose, empirical methods have been proposed to correct the fluorescence emission spectrum (Gitelson et al., 1998), but these methods provide results that differ from outputs of physically-based models (Cordon \& Lagorio, 2006, 2007a, b). Not many of these simulated fluorescence emission spectra have been actually compared to measurements, most of the time they are expressed in relative units and their spectral resolution is higher than $5 \mathrm{~nm}$. This is inadequate for top-of-atmosphere studies, particularly in the narrow oxygen absorption bands of the solar spectrum that are intensively investigated for passive ChlF emission measurements. Moreover, some of these models definitely calculate the fluorescence spectrum of a leaf but not its reflectance and transmittance, and when these are available, they are seldom validated. In consequence, there is a need for a leaf fluorescence model supported by strong physical assumptions, running with a limited number of input variables, and simulating ChlF with accuracy.

In this paper, we present a new leaf fluorescence model, FluorMODleaf, which is derived from the PROSPECT model. The first section presents the material and methods implemented to measure PSI and PSII excitation spectra. The second one details the physics of scattering, absorption and fluorescence emission within the model, as well as the fluorescence source function, i.e., the model of fluorescence emission at the chloroplast level. In the third section, a sensitivity analysis of the model and a comparison of its outputs with experimental measurements are presented. The conclusion goes back over the potentials and limits of FluorMODleaf.

\section{Materials and methods}

Intact chloroplasts were isolated from spinach (Spinacia oleracea L.) as described in Laasch (1987) and then osmotically shocked. The thylakoids obtained this way at a concentration of $1.6 \mathrm{mg} \mathrm{Chl} \mathrm{ml}^{-1}$ were diluted for fluorescence measurements. PSII and PSI samples were prepared according to Berthold et al. (1981) and Mullet et al. (1980), respectively. In order to avoid reabsorption artifacts, all fluorescence emission and excitation spectra were measured in a $1 \mathrm{~cm}$ path length quartz cuvette and diluted until they remained unchanged (final chlorophyll concentration of about 1-2 $\mu \mathrm{g} \mathrm{ml}^{-1}$ ). Fluorescence excitation spectra of thylakoids, PSI and PSII suspensions were obtained with the Cary Eclipse fluorescence spectrophotometer (Varian Inc., U.S.A.) by varying the excitation wavelength at a fixed emission wavelength of $740 \mathrm{~nm}$. The spectral resolution is $1 \mathrm{~nm}$. To correct the shape of the excitation spectra, the intensity $S(\lambda)$ of the excitation beam generated by the xenon flash-lamp of the spectrometer was measured using a calibrated photodiode (S1337-1010BQ Hamamatsu Photonics, Japan). Corrected excitation spectra were obtained by dividing the experimental spectrum by $S(\lambda)$ expressed in quantum units.

\section{Description of the model}

FluorMODleaf is the name given to the leaf fluorescence model that we designed to predict the reflectance, transmittance, upward and downward ChlF emission of a green leaf supposing that it is isolateral. It is based on PROSPECT, a radiative transfer code that pioneered the computation of the directional-hemispherical reflectance (DHR) and transmittance (DHT) of various plant leaves (monocots and dicots) over the solar spectrum, and which is now widely used in the remote sensing community (Jacquemoud \& Baret, 1990). The model mimics the leaf as a stack of several absorbing plates with rough surfaces giving rise to isotropic diffusion (Benford, 1923; Allen et al., 1970). The main advantage of PROSPECT is that $i$ ) it has few input variables, ii) absorption and scattering of light are well separated, iii) model inversion can be easily performed for calibration or validation purposes, and $i v$ ) the input variables can be either measured or estimated.

PROSPECT -5 , the very last version of the model recently published by Feret et al. (2008), needs five input variables to calculate the DHR and DHT from $400 \mathrm{~nm}$ to $2500 \mathrm{~nm}$ at a spectral resolution of $1 \mathrm{~nm}$ : the leaf structure parameter $N$ that represents the number of compact layers specifying the amount of cell wall-air space interfaces within the mesophyll, the chlorophyll $a+b$ content $C_{a b}\left(\mu \mathrm{g} \mathrm{cm}^{-2}\right)$, the total carotenoid content $C_{c x}\left(\mu \mathrm{g} \mathrm{cm}{ }^{-2}\right)$, the equivalent water thickness $C_{w}$ ( $\mathrm{g} \mathrm{cm}^{-2}$ or $\mathrm{cm}$ ), and the dry matter content $C_{m}$ also called leaf mass per area $\left(\mathrm{g} \mathrm{cm}^{-2}\right)$. Like in PROSPECT, the FluorMODleaf model considers homogeneous absorbing plates with rough surfaces, except that the intra-plate medium is fluorescent. We introduce $F_{u}$ and $F_{d}$ as the upward and downward fluorescence coefficients of the plate, respectively, and the observed fluorescence fluxes are $F_{u}^{i_{0}}$ and $F_{d}^{i_{0}}$, respectively, when the leaf is illuminated by the excitation flux $i_{0}$.

\subsection{Fluorescence of a single plate}

\subsubsection{Computation of absorbed flux in an elementary layer}

Consider a single plate illuminated by incident light $i_{0}$ that is a function of wavelength and that can be either a direct flux, a diffuse flux, or a mixture (Fig. 1). As the plate surface is rough, all fluxes reflected or transmitted at the interface of the medium are isotropic. In Fig. 1, these fluxes are designated $i_{1}, j_{1}, i_{3}$ and $j_{3}$. We define an infinitesimal horizontal layer $L(x)$ inside the plate, located at depth $x$, where $x$ refers to the distance from the top of the plate. The layer $L(x)$ receives the flux $i(x)$ traveling downward from the top of the plate $(x=0)$ and the flux $j(x)$ traveling upward from the bottom $(x=1)$. The fluorescence emission $d f\left(\lambda_{e x}, \lambda_{e m}, x\right)$ at depth $x$ is proportional to the amount of photons absorbed by the infinitesimal layer $L(x)$ and can be written as:

$d f\left(\lambda_{e x}, \lambda_{e m}, x\right)=\phi\left(\lambda_{e x}, \lambda_{e m}\right) d a\left(\lambda_{e x}, x\right)$

where $\lambda_{e x}$ and $\lambda_{e m}$ are the excitation and emission wavelengths, respectively, $\phi\left(\lambda_{e x}, \lambda_{e m}\right)$ is a source function, and $d a\left(\lambda_{e x}, x\right)$ is the absorbed flux. The source function $\phi\left(\lambda_{e x}, \lambda_{e m}\right)$ is the spectral fluorescence yield (SFY) of the elementary layer, which can be defined as the probability that one photon absorbed by the medium at $\lambda_{e x}$ be emitted at $\lambda_{e m}$. The absorbed flux $d a\left(\lambda_{e x}, x\right)$ is related to flux variations through the layer $L(x)$, therefore it can be calculated by deriving the propagating fluxes $i(x)$ and $j(x)$ with respect to $x$ :

$d a(x)=(i(x)-i(x+d x))+(j(x+d x)-j(x))=\left(-\frac{d i}{d x}(x)+\frac{d j}{d x}(x)\right) d x$. 


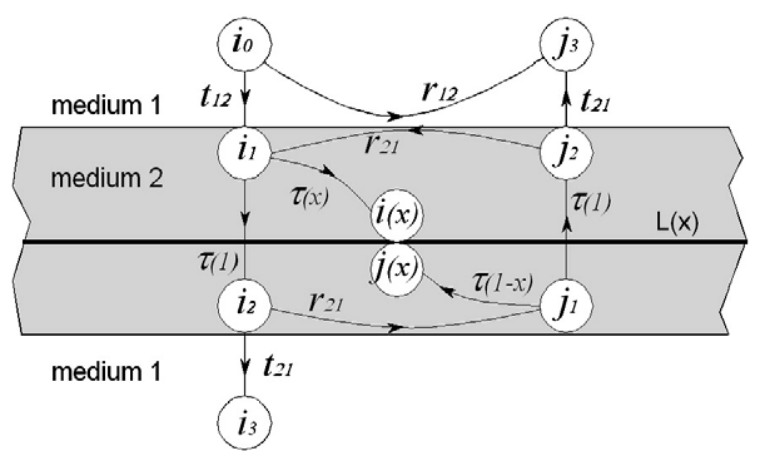

Fig. 1. Flux network in a semi-transparent plate made of an absorbing medium with rough surfaces. $i_{0}, i_{1}, i_{2}, i_{3}$ and $i(x)$ are the downward fluxes. $j_{0}, j_{1}, j_{2}$ and $j(x)$ are the upward fluxes. $i_{1}$, $i_{2}, j_{1}$ and $j_{2}$ are the internal fluxes close to the interface. $i(x)$ and $j(x)$ are incident fluxes on an infinitesimal layer $L(x) . t_{12}$ (or $t_{21}$ ) and $r_{12}$ (or $r_{21}$ ) are the transmittance and reflectance of the interface from medium 1 to 2 (or from 2 to 1 ). $\tau(x)$ is the transmittance of the inner medium for an isotropic light traveling through a section of thickness $x$.

We propose hereafter to use graphs to represent the internal fluxes within the plate. This graphical method can be applied to any algebraic problem that is intended to compute fluxes, and the appropriate system of equations is intuitively set up: the nodes represent the fluxes and the arrows stand for the physical processes through which one flux is linked with another. As an example, Fig. 1 shows all the fluxes and their interconnections inside a single plate illuminated by an incident radiation $i_{0}$. The infinitesimal layer $L(x)$ is also depicted. The flux $i_{0}$ is split in two fluxes, $i_{1}$ and $j_{3}$ : the branch from $i_{0}$ to $i_{1}$ represents the light transmitted through the interface between the air (medium 1) and the plate (medium 2) with a transmissivity (or transmission coefficient) $t_{12}$. The contribution of this branch to $i_{1}$ is then $t_{12} i_{0}$. The branch connecting $i_{0}$ to $j_{3}$ corresponds to the partial reflection at the interface between media 1 and 2 , with a reflectivity (or reflection coefficient) $r_{12}$. When several branches converge on a given node, the corresponding flux is obtained by summing all the incoming fluxes attached to them. For example, $i_{1}$ is calculated by adding the contributions of $i_{0}$ and $j_{2}$ :

$i_{1}=t_{12} i_{0}+r_{21} j_{2}$

Similar rules apply to all fluxes of the network depicted in Fig. 1. This leads to the following system of linear equations:

$$
\begin{array}{ll}
i_{1}=t_{12} i_{0}+r_{21} j_{2} & j_{1}=r_{21} i_{2} \\
i_{2}=\tau(1) i_{1} & j_{2}=\tau(1) j_{1} \\
i_{3}=t_{21} i_{2} & j_{3}=t_{21} j_{2}+r_{12} i_{0} \\
i(x)=\tau(x) i_{1} & j(x)=\tau(1-x) j_{1}
\end{array}
$$

where $r_{12}$ and $t_{12}$ have been defined earlier, and $r_{21}$ and $t_{21}$ are the reflectivity and transmissivity at the interface separating medium 2 from medium 1 . The reflectivity $r_{12}$ is calculated from the Fresnel equations as in PROSPECT (Jacquemoud \& Baret, 1990) and $t_{12}$ can be easily deduced via the relation $t_{12}=1-r_{12}$ when no absorption occurs at the interface. Remembering that $t_{21}=t_{12} / n^{2}$ avoids unnecessary calculations. The coefficient $\tau(x)$ is the transmissivity of a layer of the inner medium for isotropic radiation traveling from the surface to a level $x$ inside the plate. It can be obtained by integration of Beer's law over the hemisphere (for details, see Miller et al., 2005).

$\tau(x)=\frac{\int_{0}^{2 \pi} d \varphi \int_{0}^{\pi / 2} \tau(\theta, x) \cos \theta \sin \theta d \theta}{\int_{0}^{2 \pi} d \varphi \int_{0}^{\pi / 2} \cos \theta \sin \theta d \theta}=(1-k x) \exp (-k x)+k^{2} x^{2} \Gamma(0, k x)$

where $\theta$ and $\varphi$ are the zenith and azimuth angles of the incident flux, $k$ is the absorption coefficient of the inner medium and $\Gamma(0, k x)$ is the "upper" incomplete gamma function. By solving the system of linear Eq. (4), we get the expressions of $i(x)$ and $j(x)$ :

$$
\begin{aligned}
& i(x)=\frac{i_{0} t_{12} \tau(x)}{1-r_{21}^{2} \tau(1)^{2}} \\
& j(x)=\frac{i_{0} t_{12} r_{21} \tau(1) \tau(1-x)}{1-r_{21}^{2} \tau(1)^{2}} .
\end{aligned}
$$

Finally, $\frac{d i}{d x}(x)$ and $\frac{d j}{d x}(x)$ can be deduced by derivation of Eqs. (6) and (7) with respect to $x$, assuming that the first derivative of $\tau(x)$ is expressed by:

$\frac{d \tau}{d x}(x)=2 k^{2} x \Gamma(0, k x)-2 k \exp (-k x)$.

\subsection{2.. Computation of the fluorescence of a single plate}

The combination of Eqs. (1), (2), (5)-(7) yields the expression of the fluorescence emission of the infinitesimal layer $L(x)$ :

$d f(x)=\phi\left(\lambda_{e m}, \lambda_{e x}\right) \frac{i_{0} t_{12}\left(r_{21} \tau(1) \frac{d \tau}{d x}(1-x)-\frac{d \tau}{d x}(x)\right)}{1-r_{21}^{2} \tau(1)^{2}}$.

To calculate the fluorescence emission of the whole plate, we first have to evaluate the reabsorption of the emitted flux by the inner medium, taking into account internal reflections on the interfaces, and then to integrate the result over the whole plate for $x$ varying between 0 and 1. Once again, the problem can be approached using a network of emitted fluxes to set up the simultaneous equations describing fluorescence reabsorption (Fig. 2):

$$
\left\{\begin{array}{l}
d f_{u}=t_{f 21} d f_{u 1} \\
d f_{u 1}=\frac{1}{2} \tau_{f}(x) d f(x)+\tau_{f}(1) d f_{u 2} \\
d f_{u 2}=r_{f 21} d f_{d 2} \\
d f_{d 1}=r_{f 21} d f_{u 1} \\
d f_{d 2}=\frac{1}{2} \tau_{f}(1-x) d f(x)+\tau_{f}(1) d f_{d 1} \\
d f_{d}=t_{f 21} d f_{d 2}
\end{array}\right.
$$

where the subscript $f$ indicates that the fluorescence emission wavelength $\lambda_{e m}$ is involved. Since fluorescence is an isotropic light source, emitted photons are equally distributed between the upward

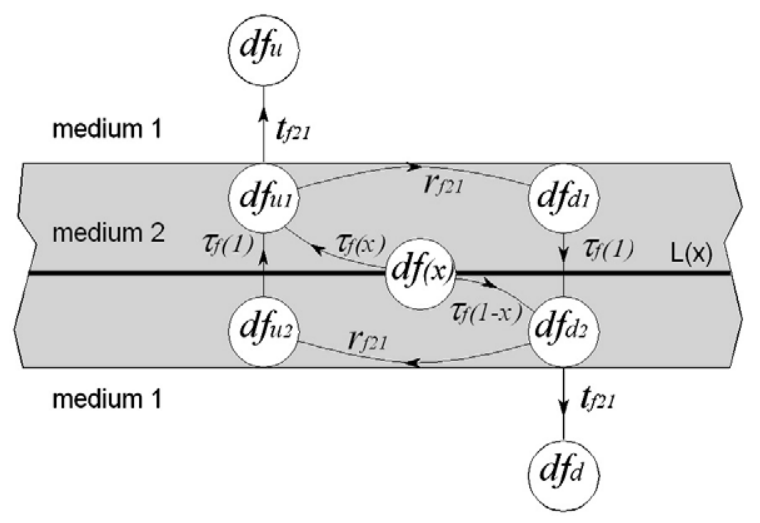

Fig. 2. Flux network of fluorescence emission of the elementary layer $L(x)$ at depth $x$. 
and downward directions and the transmission function $\tau_{f}(x)$ is simply obtained by replacing $k$ with $k_{f}$ in $\tau(x)$ (Eq. (5)). The solution of Eq. (10) gives the infinitesimal upward and downward fluorescence fluxes $d f_{u}$ and $d f_{d}$ :

$$
\left\{\begin{array}{l}
d f_{u}=\frac{t_{f 21} d f(x)\left(r_{f 21} \tau_{f}(1) \tau_{f}(1-x)+\tau_{f}(x)\right)}{2\left(1-r_{f 21}^{2} \tau_{f}(1)^{2}\right)} \\
d f_{d}=\frac{t_{f 21} d f(x)\left(\tau_{f}(1-x)+r_{f 21} \tau_{f}(1) \tau_{f}(x)\right)}{2\left(1-r_{f 21}^{2} \tau_{f}(1)^{2}\right)}
\end{array}\right.
$$

The upward and downward fluorescence signals $F_{u}(1)$ and $F_{d}(1)$ of the whole plate are then obtained as the integral of $d f_{u}$ and $d f_{d}$ from $x=0$ to $x=1$ :

$F_{u}(1)=\int_{0}^{1} d f_{u}$ and $F_{d}(1)=\int_{0}^{1} d f_{d}$.

These expressions cannot be integrated analytically so we did it numerically for a range of $k$ and $k_{f}$ values in order to build a look-up table and speed up the computation.

\subsection{Fluorescence of $N$ plates}

Consider now a stack of $N$ uniform fluorescent plates illuminated by an anisotropic incident light beam of intensity $i_{0}$, inscribed in a cone defined by its half angle $\alpha$. The output variables $R_{\alpha}(N)$ and $T_{\alpha}(N)$ are the reflectance and the transmittance of this system at the excitation wavelength $\lambda_{e x}$. The subscript $\alpha$ is removed when light is isotropic $\left(\alpha=90^{\circ}\right)$. In a similar way, we can define $F_{u}(N)$ and $F_{d}(N)$ the upward and downward apparent fluorescence yields of $N$ plates at the emission wavelength $\lambda_{e m}$. If we add a new plate at the bottom of the stack, the reflectance and transmittance become $R_{\alpha}(N+1)$ and $T_{\alpha}(N+1)$ and the upward and downward fluorescence yields $F_{u}(N+1)$ and $F_{d}(N+1)$. We can link the new system ( $N+1$ plates) to the previous one ( $N$ plates) through the network of fluxes represented in Fig. 3 and the equations below:

$$
\begin{array}{ll}
i_{1}=T_{\alpha}(N) i_{0}+R(N) j_{1} & i_{2}=T(1) i_{1}=T i_{0} \\
j_{1}=R(1) i_{1} & j_{2}=R_{\alpha}(N) i_{0}+T(N) j_{1}=R i_{0} \\
k_{1}=F_{d}(N) i_{0}+F_{u}(N) j_{1}+R_{f}(N) l_{1} & k_{2}=F_{d}(1) i_{1}+T_{f}(1) k_{1}=F_{d}(N+1) i_{0} \\
l_{1}=F_{u}(1) i_{1}+R_{f}(1) k_{1} & l_{2}=F_{u}(N) i_{0}+F_{d}(N) j_{1}+T_{f}(N) l_{1} \\
& =F_{u}(N) i_{0}
\end{array}
$$

where $R_{f}(N)$ and $T_{f}(N)$ are the reflectance and the transmittance of a pile of $N$ plates at the emission wavelength $\lambda_{e m}$. Solving Eq. (13) yields expressions of fluorescence $F_{u}(N+1)$ and $F_{d}(N+1)$ for a stack of $N+1$ plates:

$$
\begin{aligned}
F_{u}(N+1)= & F_{u}(N)+\frac{F_{d}(N) T_{\alpha}(N) R_{90}(1)}{1-R_{90}(N) R_{90}(1)}+\frac{F_{d}(N) T_{f}(N) R_{f}(1)}{1-R_{f}(N) R_{f}(1)} \\
& +\frac{T_{f}(N) T_{\alpha}(N)\left(F_{u}(1)+F_{u}(N) R_{f}(1) R_{90}(1)\right)}{\left(1-R_{90}(N) R_{90}(1)\right)\left(1-R_{f}(N) R_{f}(1)\right)} \\
F_{d}(N+1)= & \frac{F_{d}(1) T_{\alpha}(N)}{1-R_{90}(N) R_{90}(1)}+\frac{F_{d}(N) T_{f}(1)}{1-R_{f}(N) R_{f}(1)} \\
& +\frac{T_{f}(1) T_{\alpha}(N)\left(F_{u}(1) R_{f}(N)+F_{u}(N) R_{90}(1)\right)}{\left(1-R_{90}(N) R_{90}(1)\right)\left(1-R_{f}(N) R_{f}(1)\right)} .
\end{aligned}
$$

Since the upward fluorescence $F_{u}(1)$ and downward fluorescence $F_{d}(1)$ of a single layer have been calculated earlier in Eq. (12), it is possible to recursively compute these quantities for $2,3, \ldots N$ layers, with $N$ a positive integer, and then to extend the calculation to a real number by interpolation.

\subsection{The fluorescence source model}

In Eq. (1), we have introduced the spectral fluorescence yield (SFY) of the elementary layer $\phi\left(\lambda_{e x}, \lambda_{e m}\right)$. In the case of a single fluorophore, it can be written as:

$\phi\left(\lambda_{e x}, \lambda_{e m}\right)=\xi\left(\lambda_{e x}\right) Q \eta\left(\lambda_{e m}\right)$

with $\xi\left(\lambda_{e x}\right)$ the excitation efficiency spectrum, $Q$ the fluorescence quantum yield, and $\eta\left(\lambda_{e m}\right)$ the fluorescence emission spectrum normalized to unity so that $\int_{0}^{\infty} \eta\left(\lambda_{e m}\right) d \lambda_{e m}=1$. Details for these three terms are provided below. In photosynthetic systems such as plant leaves, red fluorescence is only produced by chlorophyll $a$ but it is well-known that other pigment-protein complexes are involved in fluorescence emission with different spectral and efficiency characteristics. The chlorophylls associated with the photosystems I (PSI) and II (PSII) are the two main fluorophores of plant leaves (Emerson et al., 1957). Carotenoids also play a crucial role in light absorption and partial energy transfer to chlorophylls (Duysens, 1952). We therefore split the source function in two terms, one for each photosystem, and rewrite Eq. (16) as:

$$
\phi\left(\lambda_{e x}, \lambda_{e m}\right)=\sigma_{I} \xi_{I}\left(\lambda_{e x}\right) Q_{I} \eta_{I}\left(\lambda_{e m}\right)+\sigma_{I I} \xi_{I I}\left(\lambda_{e x}\right) Q_{I I} \eta_{I I}\left(\lambda_{e m}\right)
$$

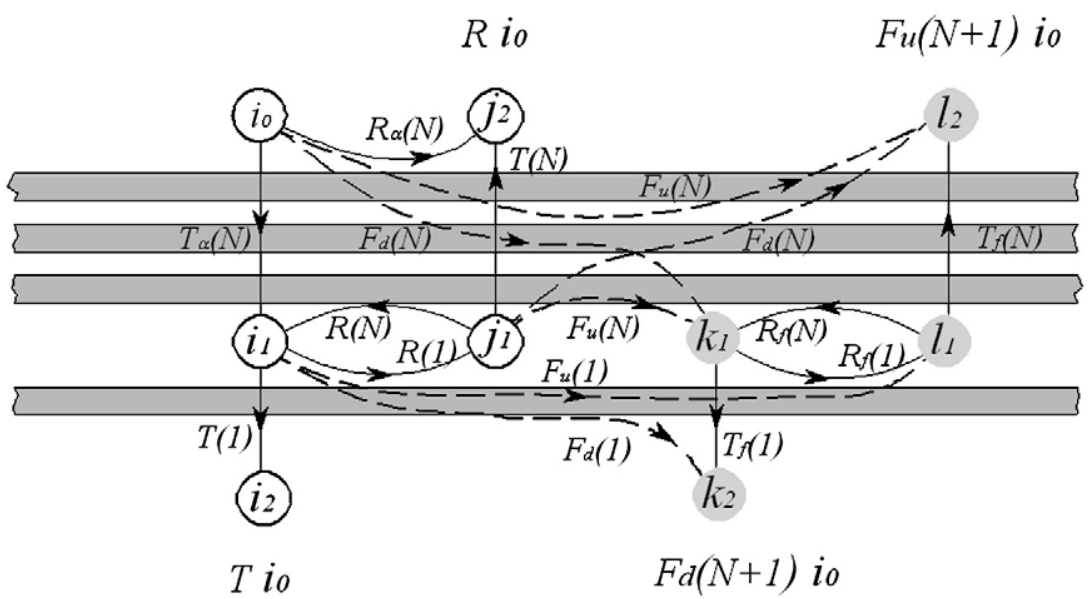

Fig. 3. Flux network of excitation light and fluorescence emission in a pile of $N+1$ plates. $i_{0}, i_{1}$ and $i_{2}$ are the downward incident fluxes. $j_{1}$ and $j_{2}$ are the upward incident fluxes. $k_{1}$ and $k_{2}$ are the downward fluorescent fluxes. $l_{1}$ and $l_{2}$ are the upward fluorescent fluxes. Solid line: excitation fluxes, dashed line: emission fluxes. 
where the subscripts I and II stand for photosystems I and II, respectively. The stoichiometric factors $\sigma_{I}$ and $\sigma_{I I}$ satisfying the relation $\sigma_{I}+\sigma_{I I}=1$ are the relative absorption cross section of PSI and PSII. These constitutive parameters vary with plant species and growth light intensity and quality (Melis \& Brown, 1980; Leong \& Anderson, 1984).

\subsubsection{Excitation efficiency}

The excitation efficiency $\xi\left(\lambda_{e x}\right)$ is the fraction of photons absorbed by or transferred to Chl $a$, among all the absorbed photons that are already quantified in Eq. (2). We introduce this factor at that point because the medium may contain non-fluorescing molecules, or molecules that emit light in another wavelength domain. In plant leaves, this is the case of anthocyanins that absorb light in the blue and the green (Gitelson et al., 2001) or polyphenols that may also produce blue fluorescence when excited by ultraviolet radiation (Cerovic et al., 1999). Carotenoids also belong to this category because they partially transfer their energy to chlorophylls. If we consider an elementary leaf layer illuminated by a collimated beam of light having a uniform flux intensity $I_{0}\left(\lambda_{e x}\right)$, its fluorescence is:

$f\left(\lambda_{e x}, \lambda_{e m}\right)=I_{0}\left(\lambda_{e x}\right) k\left(\lambda_{e x}\right)\left(\sigma_{I} \eta_{I}\left(\lambda_{e m}\right) Q_{I} \xi_{I}\left(\lambda_{e x}\right)+\sigma_{I I} \eta_{I I}\left(\lambda_{e m}\right) Q_{I I} \xi_{I I}\left(\lambda_{e x}\right)\right)$

with $k\left(\lambda_{e x}\right)$ the absorption coefficient of PROSPECT. The excitation efficiency of PSI and PSII can be then expressed as:

$\xi_{I}\left(\lambda_{e x}\right)=\frac{\chi_{I}\left(\lambda_{e x}\right)}{k\left(\lambda_{e x}\right)}$

$\xi_{I I}\left(\lambda_{e x}\right)=\frac{\chi_{I I}\left(\lambda_{e x}\right)}{k\left(\lambda_{e x}\right)}$

where $\chi_{I}\left(\lambda_{e x}\right)$ and $\chi_{I I}\left(\lambda_{e x}\right)$ are the number of photons absorbed by the fluorescent pigments of photosystems $I$ and $I I$, respectively. In that case fluorescence is directly related to $\chi_{I}\left(\lambda_{e x}\right)$ and $\chi_{I I}\left(\lambda_{e x}\right)$ :

$f\left(\lambda_{e x}, \lambda_{e m}\right)=I_{0}\left(\lambda_{e x}\right)\left(\sigma_{I} \chi_{I}\left(\lambda_{e x}\right) Q_{I} \eta_{I}\left(\lambda_{e m}\right)+\sigma_{I I} \chi_{I I}\left(\lambda_{e x}\right) Q_{I I} \eta_{I I}\left(\lambda_{e m}\right)\right)$.

We experimentally assessed $\chi_{I}\left(\lambda_{e x}\right)$ and $\chi_{I I}\left(\lambda_{e x}\right)$ using dilute solutions of PSI and PSII, which are comparable to an infinitesimal layer because very little scattering occurs in such a medium. By choosing $\left(\sigma_{I}, \sigma_{I I}\right)=(1,0)$ (pure PSI fraction) or $\left(\sigma_{I}, \sigma_{I I}\right)=(0,1)$ (pure PSII fraction), and by varying the excitation wavelength while fixing the emission wavelength at $740 \mathrm{~nm}$ where reabsorption is very low, we measured the relative excitation spectra $\chi_{I}\left(\lambda_{e x}\right)$ and $\chi_{I I}\left(\lambda_{e x}\right)$. Then we normalized them so that their value near the main absorption peak at $660 \mathrm{~nm}$ is at the same level as $k_{a b}\left(\lambda_{e x}\right)$, the specific absorption coefficient of chlorophyll provided by PROSPECT-5 (Feret et al., 2008).

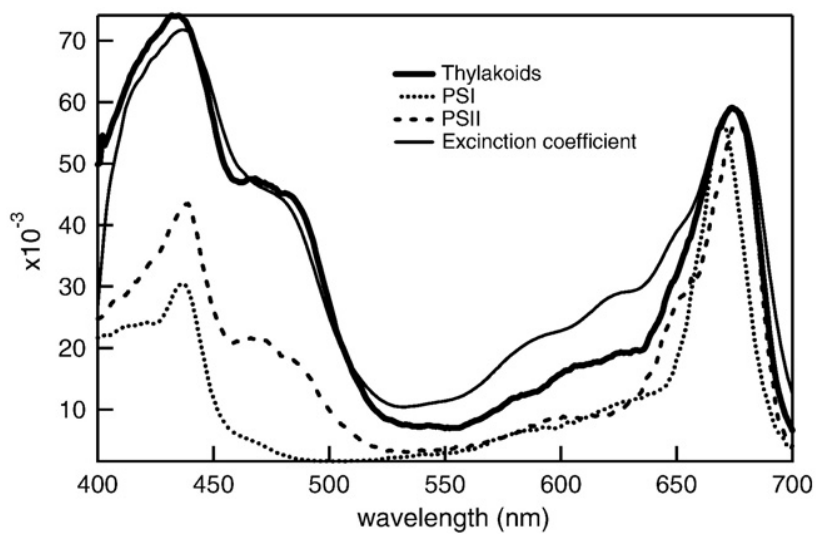

Fig. 4. Excitation spectra of thylakoids, PSI and PSII suspensions compared to the chlorophyll $a+b$ specific absorption coefficient of PROSPECT-5 (Feret et al., 2008).
This is justified because chlorophylls $a$ and $b$ are the only absorbers in this region of the visible spectrum. Fig. 4 shows the normalized excitation spectra of PSI and PSII, compared to the excitation spectrum of entire thylakoids normalized the same way and to $k_{a b}\left(\lambda_{e x}\right)$. On the one hand, it turns out that the thylakoid spectrum cannot be considered as a combination of measured PSI and PSII excitation spectra, as it was to be expected. Furthermore, the PSI excitation spectrum at $700 \mathrm{~nm}$ is generally higher than the PSII excitation spectrum (Franck et al., 2002). These anomalies may be due to conformational changes of the pigment-protein complexes: it is a well-known fact that the extraction procedure modifies the interactions between pigments. Moreover the spectroscopic properties and aggregation state of purified LHC depend on the detergent concentration in the solvent (Bassi et al., 1991; Moya et al., 2001). On the other hand, the thylakoid fluorescence excitation spectrum $\chi_{\text {thy }}\left(\lambda_{e x}\right)$ looks very similar to $k_{a b}\left(\lambda_{e x}\right)$ as seen in Fig. 4. Therefore, we propose to use it as the excitation spectrum for both photosystems, so that, by combining Eqs. (17) and (20), the source function becomes:

$\phi\left(\lambda_{e x}, \lambda_{e m}\right)=\frac{\chi_{t h y}\left(\lambda_{e x}\right)}{k\left(\lambda_{e x}\right)}\left(\sigma_{I} Q_{I} \eta_{I}\left(\lambda_{e m}\right)+\sigma_{I I} Q_{I I} \eta_{I I}\left(\lambda_{e m}\right)\right)$.

\subsubsection{Fluorescence quantum yield}

Among all the photons absorbed by $\mathrm{Chl} a$, a small fraction $Q$ called fluorescence quantum yield is reemitted by fluorescence. Another important parameter is the fluorescence lifetime $\tau$ defined as the average time the molecule stays in its excited state before emitting a photon. The quantities $Q$ and $\tau$ are connected by the relation $Q=\frac{\tau}{\tau_{0}}$ where $\tau_{0}$ is the natural fluorescence lifetime of Chl $a$ when fluorescence is the only de-excitation pathway. The natural lifetime $\tau_{0}$ is an intrinsic property of this pigment whether it is involved in PSI or PSII. It is calculated by integration of the absorption curve and is equal to about 15 ns (Brody \& Rabinowitch, 1957). The fluorescence lifetime of isolated PSI complexes $\left(\tau_{I}\right)$ has long been recognized to be very short: it was found to be about 0.03 ns (Borisov \& Il'ina, 1973) and 0.1 ns (Schmuck \& Moya, 1994; Agati et al., 2000). Measurements on isolated PSI complexes extracted from Synechoccocus elongatus revealed that the PSI fluorescence decay displayed a fast component (13 ps) and a dominant component ( 95\% of the decay amplitude at the maximum) with lifetimes ranging from $34 \mathrm{ps}$ for open centers to $37 \mathrm{ps}$ for closed centers (Byrdin et al., 2000). This weak variation of $\tau_{I}$ supports the assumption that unlike PSII, PSI fluorescence does not

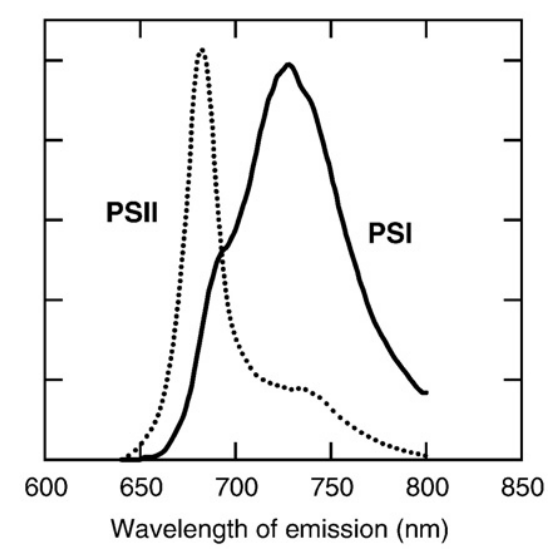

Fig. 5. Normalized elementary emission spectra $\eta\left(\lambda_{e m}\right)$ of isolated PSI (straight line) and PSII (dotted line) in dilute suspension, measured before reabsorption. PSI: PSI-LHCI preparation from maize (Zea mays) in a $3 \mathrm{~mm} \times 3 \mathrm{~mm}$ cuvette at an optical density of 0.03, excitation wavelength $475 \mathrm{~nm}$, temperature $280 \mathrm{~K}$ (Croce et al., 1996). PSII: PSII particles from barley (Hordeum vulgare) in a $1 \mathrm{~mm}$ cuvette, broad band excitation provided by a white light source filtered with a combination of filters (maximum transmission $446 \mathrm{~nm}$, half-band-width $90 \mathrm{~nm}$ ) (Franck et al., 2002). 
Table 1

Range and standard values of FluorMODleaf input variables.

\begin{tabular}{|c|c|c|c|c|c|}
\hline Variable & Symbol & Range & Standard value & Unit & References \\
\hline Leaf structure parameter & $N$ & $1-2.5$ & 1.5 & - & Jacquemoud and Baret (1990) \\
\hline Chlorophyll $a+b$ content & $C_{a b}$ & $0.4-76.8$ & 33.0 & $\mu \mathrm{g} \mathrm{cm}{ }^{-2}$ & Feret et al. (2008) \\
\hline Total carotenoid content & $C_{c x}$ & $0-25.3$ & 8.0 & $\mu \mathrm{g} \mathrm{cm}-2$ & Feret et al. (2008) \\
\hline Water content & $C_{w}$ & $0.0044-0.0340$ & 0.01 & $\mathrm{~cm}$ & Feret et al. (2008) \\
\hline Dry matter content & $C_{m}$ & $0.0017-0.0331$ & 0.005 & $\mathrm{~g} \mathrm{~cm}^{-2}$ & Feret et al. (2008) \\
\hline Relative absorption cross section ratio & $\sigma_{I I} / \sigma_{I}$ & $1.0-2.4$ & 1.0 & - & $\begin{array}{l}\text { Anderson and Melis (1983), Yamazaki et al. (1999), } \\
\text { Lokstein et al. (2002), Danielsson et al. (2004) }\end{array}$ \\
\hline PSI fluorescence lifetime & $\tau_{I}$ & $0.034-0.1$ & 0.035 & ns & $\begin{array}{l}\text { Borisov and Il'ina (1973), Schmuck and Moya (1994), } \\
\text { Agati et al. (2000), Byrdin et al. (2000) }\end{array}$ \\
\hline PSII fluorescence lifetime & $\tau_{I I}$ & $0.3-2.0$ & 0.5 & ns & Schmuck et al. (1991) \\
\hline
\end{tabular}

change with photochemistry. In the following, we set $\tau_{I}=0.035 \mathrm{~ns}$ as a standard value of PSI fluorescence lifetime, although it could be species dependent, which would explain that some authors found higher values. The fluorescence lifetime of PSII $\left(\tau_{I I}\right)$ is much longer than $\tau_{I}$ (Hodges \& Moya, 1988), so lifetime measurements on chloroplasts or leaves at $685 \mathrm{~nm}$ represent a good approximation of the lifetime of isolated PSII. The PSII quantum yield $Q_{I I}$ is known to produce variable chlorophyll fluorescence under the influence of photochemical (closure of the reaction centers) and non-photochemical quenching, and consequently to reflect the physiological state of the plant (for a comprehensive review, see Krause \& Weis, 1991). Although several components are involved in the PSII fluorescence emission (Moya et al., 1986), it is long established that the mean fluorescence lifetime $t_{I I}$ is almost linearly related to $Q_{I I}$ in most experimental conditions (Tumerman \& Sorokin, 1967; Moya, 1974). The near-linearity of $\tau_{I I}$ versus $Q_{I I}$ experimentally observed results from energy migration within the photosynthetic antennae pigments.
Thus, the quantum yield $Q_{I I}$ can be modeled by the simple relation $Q_{I I}=\frac{\tau_{I I}}{\tau_{0}}$ too. Time-resolved fluorescence measurements on leaves showed that $\tau_{I I}$ varied from $0.3 \mathrm{~ns}$ in the $F_{0}$ state (Goulas, 1992) to about 2 ns in the $F_{m}$ state (Schmuck et al., 1991), and that its value was about $0.5 \mathrm{~ns}$ in the $F_{S}$ state (Schmuck et al., 1991). Under natural conditions, the stationary fluorescence $F_{S}$ stays in the range between $F_{0}$ to twice $F_{0}$ (Flexas et al., 2000). So we defined the standard conditions for our model by considering lifetimes of 0.035 ns for PSI and 0.5 ns for PSII, which gives:

$Q_{I}=\frac{\tau_{I}}{\tau_{0}}=\frac{0.035}{15}=0.23 \%$ and $Q_{I I}=\frac{\tau_{I I}}{\tau_{0}}=\frac{0.5}{15}=3.3 \%$.

\subsubsection{Fluorescence emission spectrum}

Fig. 5 shows the native PSI and PSII fluorescence emission spectra that we used in FluorMODleaf (Pedrós et al., 2008). The PSI emission spectrum displays a single broad band at $740 \mathrm{~nm}$ (Croce et al., 1996)
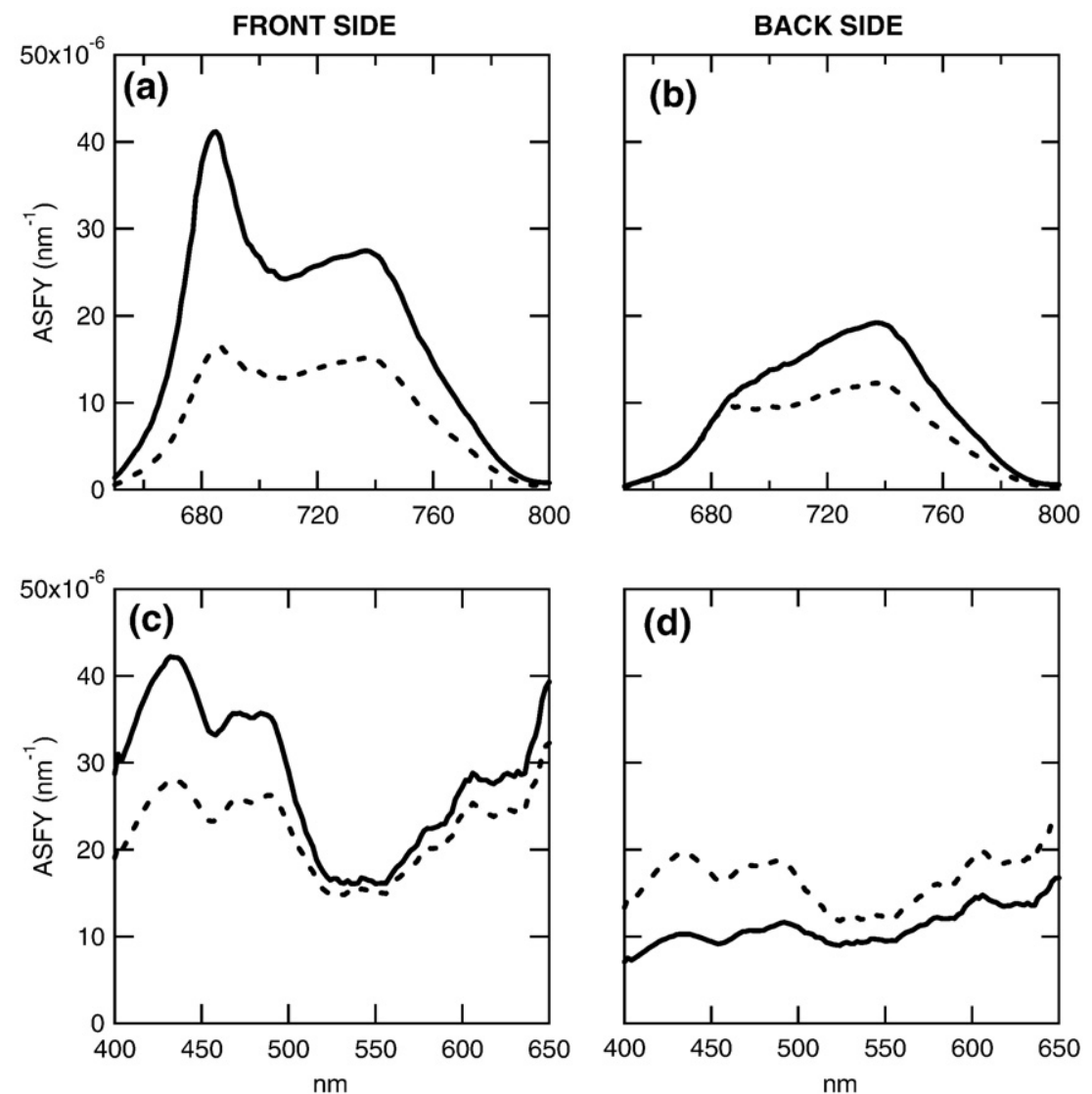

Fig. 6. Apparent spectral fluorescence yield (ASFY) of the standard leaf defined in Table 1. (a-b) Emission spectra at $\lambda_{e x}=440 \mathrm{~nm}$ (line) and $\lambda_{e x}=535 \mathrm{~nm}$ (dash); (c-d) Excitation spectra at $\lambda_{e m}=685 \mathrm{~nm}$ (line) and $\lambda_{e m}=735 \mathrm{~nm}$ (dash). 
while the main peak of the PSII emission spectrum is at $683 \mathrm{~nm}$, with a shoulder between $700 \mathrm{~nm}$ and $750 \mathrm{~nm}$ (Franck et al., 2002).

\subsubsection{Source function}

Combining Eqs. (22) and (23) allows us to write the expression of the source function:

$\phi\left(\lambda_{e x}, \lambda_{e m}\right)=\frac{\chi_{t h y}\left(\lambda_{e x}\right)}{\tau_{0} k\left(\lambda_{e x}\right)}\left(\sigma_{I} \tau_{I} \eta_{I}\left(\lambda_{e m}\right)+\sigma_{I I} \tau_{I I} \eta_{I I}\left(\lambda_{e m}\right)\right)$

\section{Model runs and discussion}

\subsection{Definition of a standard leaf}

The eight input variables of FluorMODleaf include the five basic variables of PROSPECT-5 (leaf structure parameter $N$, chlorophyll $a+b$ content $C_{a b}$, total carotenoid content $C_{c x}$, equivalent water thickness $C_{w}$ and dry matter content $C_{m}$ ) and the three new ones introduced in this paper to control fluorescence emission $\left(\sigma_{I I} / \sigma_{I}, \tau_{I}\right.$ and $\left.\tau_{I I}\right)$. We determined the range of variation of each of these input variables on the basis of published data and defined standard values to be used in the following model runs (Table 1). According to Jacquemoud and Baret (1990), the leaf structure parameter $N$ roughly ranges from 1 to 1.5 for monocots, and from 1.5 to 2.5 for dicots, with an average value of $N=1.5$. The four independent data sets that helped to calibrate and validate PROSPECT-5 (Feret et al., 2008) show consistent statistical distributions for pigments, water and dry matter. Therefore, Table 1 only displays standard values of $N, C_{a b}, C_{c x}$ and $C_{m}$ extracted from the
ANGERS data set acquired in Angers (France) and containing 276 leaf samples. We determined the standard values of $N, C_{a b}, C_{c x}, C_{w}$ and $C_{m}$ as the mean values of these parameters in the ANGERS dataset. The stoichiometric coefficients $\sigma_{I}$ and $\sigma_{I I}$ represent the relative absorption cross section of the two photosystems and quantify the relative distribution of light between them. They depend on the concentration of photosystems I and II reaction centers, their distribution in the thylakoids, antenna size and pigment composition, all factors that vary with environmental conditions (Melis, 1989). Plants tend to acclimate to these conditions in order to balance linear electron flow between the two photosystems (Chow et al., 1990). We took the ratio $\sigma_{I I} / \sigma_{I}=1$ as a standard value for this parameter. If we disregard the effect of pigment composition changes between the two photosystems, $\sigma_{I I} / \sigma_{I}$ can be approximated by the product of the PSII/PSI ratio by the antenna size. The range of variation of this product (Table 1) can be retrieved from the literature (Anderson \& Melis, 1983; Yamazaki et al., 1999; Lokstein et al., 2002; Danielsson et al., 2004).

\subsection{Emission and excitation spectra}

We calculated the apparent spectral fluorescence yield (ASFY) of the standard leaf defined in Table 1. Louis et al. (2006) defined it as the ratio of the number of photons emitted by the leaf surface, per unit spectral bandwidth, to the number of incident photons. ASFY is equivalent of the traditional fluorescence emission spectrum expressed as a number of photons but in appropriate units so that its integral over wavelength equals the Apparent Fluorescence Yield (AFY). In this paper, ASFY is expressed in $\mathrm{nm}^{-1}$. Fig. $6 \mathrm{a}$ and $\mathrm{b}$ displays emission spectra at $\lambda_{e x}=$ $440 \mathrm{~nm}$ and $\lambda_{e x}=535 \mathrm{~nm}$, two excitation wavelengths characteristic of
FRONT SIDE
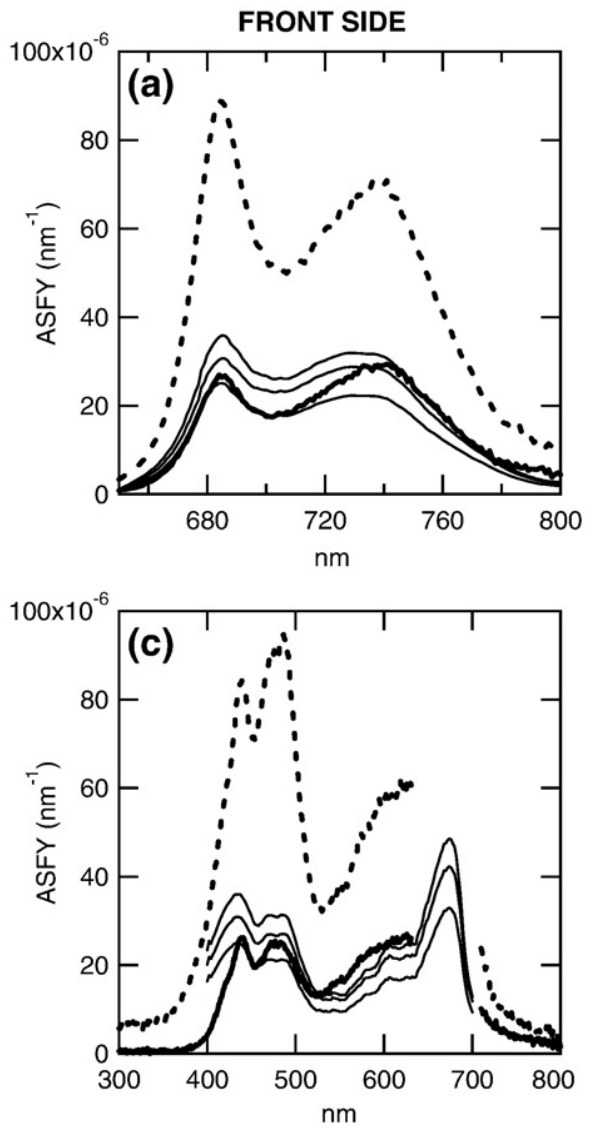

BACK SIDE
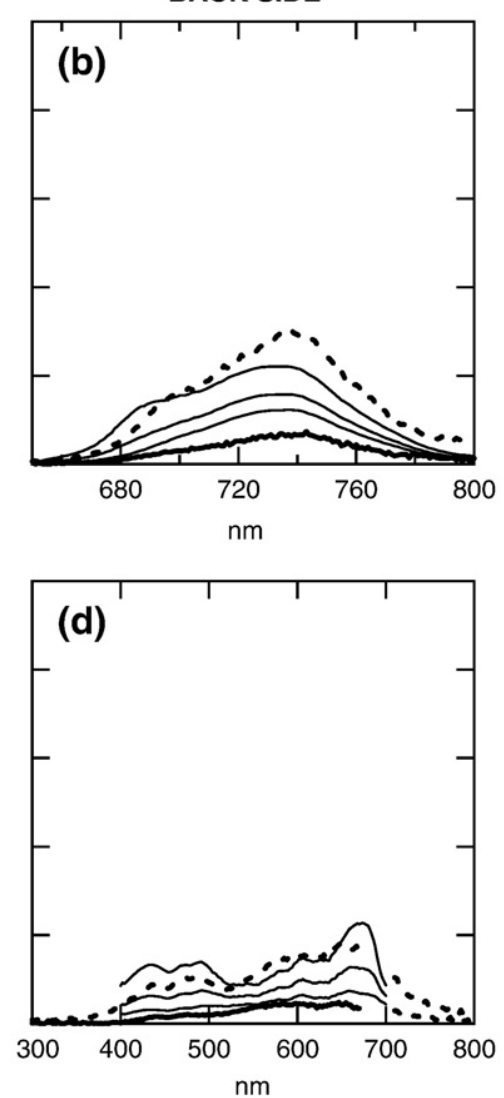

Fig. 7. Apparent spectral fluorescence yield (ASFY) computed by FluorMODleaf compared to measurements acquired on a bean leaf (Louis et al., 2006). Top (a-b): emission spectra at an excitation wavelength of $440 \mathrm{~nm}$. Bottom (c-d): excitation spectra at an emission wavelength of $687 \mathrm{~nm}$. Left (a-c): front-side configuration. Right (b-d): back-side configuration. Thick solid line: the adaxial surface is illuminated. Thick dashed line: the abaxial surface is illuminated. Thin lines: FluorMODleaf simulations with increasing values of the leaf structure parameter $N$ (bottom curve: $N=1$, middle curve: $N=2$, top curve: $N=3$ ). The other parameters are defined in Table 1, except $C_{a b}\left(24 \mu \mathrm{g} \mathrm{cm}{ }^{-2}\right)$ and $C_{c x}\left(6 \mu \mathrm{g} \mathrm{cm}^{-2}\right)$ that are taken in Louis et al. (2006). $\tau_{1}=0.1 \mathrm{~ns}$ and $\tau_{I I}=0.3 \mathrm{~ns}\left(F_{0}\right.$ state). 
high and medium pigment absorption regions. Fig. $6 \mathrm{c}$ and d displays excitation spectra at $\lambda_{e m}=685 \mathrm{~nm}$ and $\lambda_{e m}=735 \mathrm{~nm}$, two emission wavelengths aligned with the two main fluorescence emission peaks. These spectra correspond to two different measurement configurations: front-side and back-side that are the situations where fluorescence is measured on the same side and on the opposite side of the light source, respectively. ASFY is about 20 to $40 \times 10^{-6} \mathrm{~nm}^{-1}$ in the front-side configuration and 10 to $20 \times 10^{-6} \mathrm{~nm}^{-1}$ in the back-side configuration, depending on emission and excitation wavelengths. In the front-side configuration, changing the excitation wavelength from $440 \mathrm{~nm}$ to $535 \mathrm{~nm}$ induces an overall decrease of ChlF emission and a decrease of the red to far-red fluorescence ratio (RF/FRF) (Fig. 6a). This can be interpreted as a stronger reabsorption of the $535 \mathrm{~nm}$ induced fluorescence because green radiation penetrates deeper inside the leaf tissue than blue or red radiation (Vogelmann, 1989), resulting in a longer optical path length (OPL) of fluorescence emission. Switching from front-side to back- side also results in stronger fluorescence reabsorption, as illustrated by the lower overall fluorescence emission and the very low RF/FRF ratio (Fig. 6b).

Very few data have been published on absolute fluorescence yield of leaves. Louis et al. (2006) measured ASFY on dorsiventral bean (Phaseolus vulgaris L.) leaves. They found that fluorescence was much higher when the leaves were illuminated on the abaxial side than on the adaxial side, whatever the measurement geometry (Fig. 7). The RF/FRF ratio is also greater on the abaxial side. FluorMODleaf, which assumes that leaves are symmetric, is maybe not the most appropriate tool to simulate the fluorescence of such plant leaves. However, we compared experimental data with FluorMODleaf simulations obtained by varying the input variables to reproduce changes induced by turning the leaf blade upside down. Fig. 7a and c shows that FluorMODleaf reproduces quite well the emission and excitation spectra measured on the adaxial surface in the front-side configuration. However, it fails in simulating the high
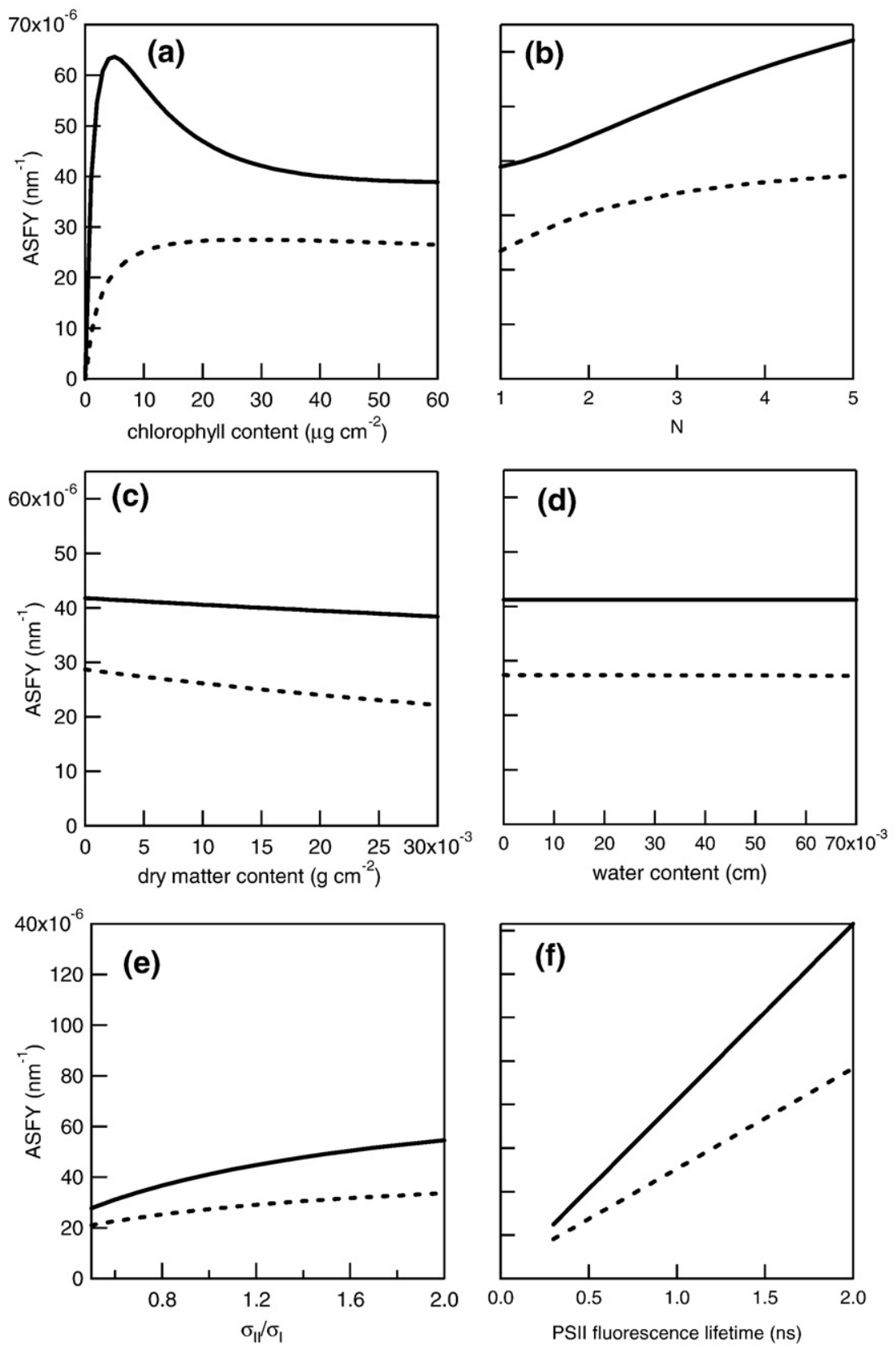

Fig. 8. Fluorescence emission as a function of (a) chlorophyll $a+b$ content, (b) leaf structure parameter, (c) dry matter content, (d) water content, (e) $\sigma_{I I} / \sigma_{I}$ ratio, and (f) PSII lifetime $\tau_{I I}$. Solid line: emission at $685 \mathrm{~nm}$, dashed line: emission at $735 \mathrm{~nm}$. The other parameters are defined in Table 1. 
fluorescence levels observed on the abaxial surface when the leaf structure parameter $N$ varies from 1 to 3 (Fig. $7 \mathrm{a}$ and c) or when the other input variables, including Chl content, vary across a physiologically acceptable range (data not shown). This is not the case in the back-side configuration, where an increase of $N$ from 1 to 3 reproduces more accurately the variation of fluorescence observed when shifting from adaxial to abaxial excitation (Fig. 7b and d). The palisade and spongy mesophylls of dicot leaves display very different anatomical structures. For instance, the number of air-cell interfaces is much higher in the spongy mesophyll. Scattering of excitation light and fluorescence is consequently enhanced in this tissue, resulting in increased OPL and greater absorption near the surface. It implies a stronger fluorescence emission with a low reabsorption, as indicated by the higher RF/FRF ratio of the abaxial surface. Indeed, increasing the leaf structure parameter $N$ amounts to shifting from adaxial to abaxial surface, but to a smaller extent. Several hypotheses can be formed to explain the discrepancy between abaxial fluorescence simulated by FluorMODleaf and experimentally measured as in Louis et al. (2006): actual scattering in the spongy mesophyll is much higher than modeled by FluorMODleaf, in particular lateral scattering that may occur to a greater extent; actinic effects change fluorescence when turning the leaf upside down. An improvement of the FluorMODleaf model including leaf asymmetry, as well as complementary measurements, are necessary to better understand side effects in the case of dicot leaves.

\subsection{Sensitivity study}

Fig. 8 presents variations of fluorescence emission in the front-side configuration, at $685 \mathrm{~nm}$ and $735 \mathrm{~nm}$, as a function of six input variables of FluorMODleaf: $C_{a b}, N, C_{m}, C_{w}, \sigma_{I I} / \sigma_{I}$ and $\tau_{I I}$. The largest variation of ChIF is obtained with photosynthetic pigments (Fig. 8a). Fluorescence emissions F685 and F735 steeply increase for low $C_{a b}$ values, but F685 interestingly decreases for $C_{a b}>5 \mu \mathrm{g} \mathrm{cm}^{-2}$ while F735 remains almost constant above $20 \mu \mathrm{g} \mathrm{cm} \mathrm{cm}^{-2}$. At first sight, such a decrease is surprising because one would expect to have more fluorescence when more fluorophore is present. Nevertheless, this effect has already been reported in the literature (Gitelson et al., 1998; Louis, 2004) and is interpreted as an increase of fluorescence reabsorption in the red with increasing chlorophyll content. Additional simulations described hereafter show that scattering plays a major role in the decrease of $\mathrm{ChlF}$ with chlorophyll content. Fluorescence emission increases with the leaf structure parameter $N$ both at $685 \mathrm{~nm}$ and $735 \mathrm{~nm}$ (Fig. 8b). Stacking elementary layers tends to increase multiple scattering and then to lengthen the optical path of light. It could explain the greater absorption of excitation light when $N$ increases and also why fluorescence emission from the abaxial surface is greater than from the adaxial surface (Louis et al., 2006). This was already observed by Virgin (1954) who studied the effect of water infiltration of plant leaves on their fluorescence spectrum. Internal scattering is greatly reduced due to a change in the air volume ratio in the mesophylls. The abaxial part of the mesophyll that contains more intercellular spaces is consequently more diffusive, which can be interpreted by a higher $N$ value. ASFY slightly depends on $C_{m}$ with a variation of $8 \%$ at $685 \mathrm{~nm}$ over the range of dry matter content reported in the ANGERS database (Fig. 8c). Water does not absorb visible light so that fluorescence emission does not depend on $C_{w}$ (Fig. 8d). Finally, fluorescence also increases with $\sigma_{I I} / \sigma_{I}$ ratio and $\tau_{I I}$ (Fig. 8e and f). In both cases, one can notice that the increase in fluorescence is greater at $685 \mathrm{~nm}$ than at $735 \mathrm{~nm}$, because the contribution of photosystem II to fluorescence emission is greater in the red than in the far-red. In FluorMODleaf, the quenching state of PSII is represented by $\tau_{I I}$ which is under the influence of photochemical and non-photochemical quenching. Therefore physiological models should compute the fluorescence quantum yield as a function of environmental parameters and physiological status of the plant, and link it to FluorMODleaf through this parameter.

\subsection{Red/far-red fluorescence ratio}

It is generally recognized that the RF/FRF ratio decreases when the chlorophyll content increases (for a comprehensive review, see Buschmann, 2007). As the chlorophyll absorption bands overlap the red but not the far-red emission bands, this effect has been attributed to the reabsorption of red light emitted at $685 \mathrm{~nm}$ by chlorophyll itself. Fig. 9 illustrates the variation of the ratio F685/F735 with $C_{a b}$ : FluorMODleaf simulations entirely agree with experimental data (Gitelson et al., 1998).

\subsection{Effect of scattering on fluorescence emission}

Multiple scattering is known to play an important role on light propagation in leaves, and therefore on fluorescence. When modeling fluorescence emission using the KM theory, for instance, it is however difficult to separate absorption from scattering, and extinction coefficients tend to mix these two effects. Some models introduce a factor $\beta$ that artificially increases the actual extinction coefficient of chlorophyll to account for scattering within the leaf (Agati et al., 1993). In FluorMODleaf, the plate medium itself is supposed to be non-diffusive, so that scattering is only caused by internal reflections at the plate interfaces. It consequently only depends on the refractive index of leaf materials and the number of plates $N$. We modeled pure scattering effects by varying solely the $N$ parameter (Fig. $8 \mathrm{~b}$ ) and removed them completely by setting all refractive indexes to 1 . Variation of ChlF emission as a function of $C_{a b}$ has been computed in the front-side and back-side configurations at two wavelengths: $685 \mathrm{~nm}$ where a significant decrease of ChlF is observed in both cases (Fig. 10a) and $735 \mathrm{~nm}$ where it is very low (Fig. 10c). When diffusive effects are removed in FluorMODleaf (Fig. 10b and d), the decrease of the front-side ChlF at $685 \mathrm{~nm}$ is no longer observed, while ChlF at $735 \mathrm{~nm}$ remains almost unchanged. This corresponds to a decrease of fluorescence reabsorption at $685 \mathrm{~nm}$. Indeed, scattering is known to lengthen the optical pathway inside the leaf, thus increasing reabsorption in the red. On the other hand, front-side fluorescence at $685 \mathrm{~nm}$ without scattering does not show any decrease with $C_{a b}$ (Fig. 10b) while back-side fluorescence still tends to zero for high chlorophyll contents. This demonstrates that scattering plays a crucial role in the variation of ChlF at $685 \mathrm{~nm}$ in the front-side configuration by reflecting part of the fluorescence flux emitted by the deeper layers of the leaf toward the most illuminated ones. (Fig. 10a). Conversely, scattering does not seem to play an important role in the $735 \mathrm{~nm}$ emission where reabsorption is low as shown in Fig. 10c and d.

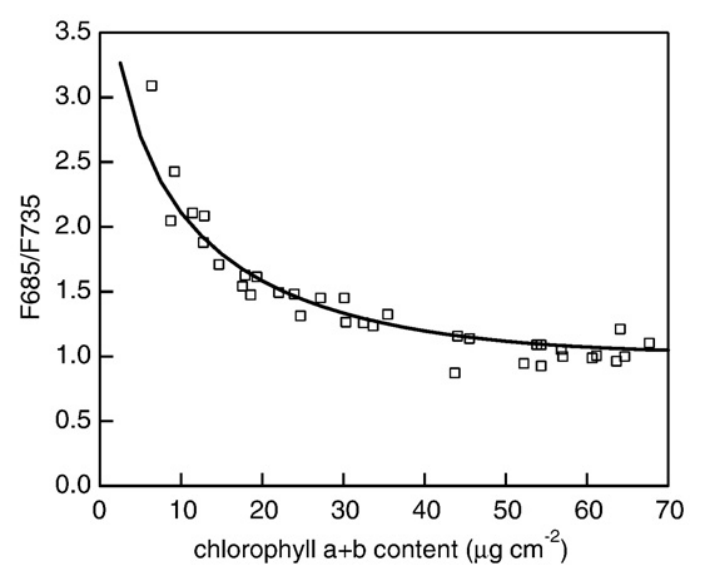

Fig. 9. Red to far-red fluorescence ratio F685/F735 as a function of chlorophyll $a+b$ content for $\lambda_{e x}=430 \mathrm{~nm} . N=3, \sigma_{I I} / \sigma_{I}=0.8$ and $\tau_{I I}=0 . \mathrm{ns}$. The other parameters are defined in Table 1. Solid line: model output. Squares: data taken from Gitelson et al. (1998) and obtained on elm leaves at the same excitation wavelength. 
WITH DIFFUSION
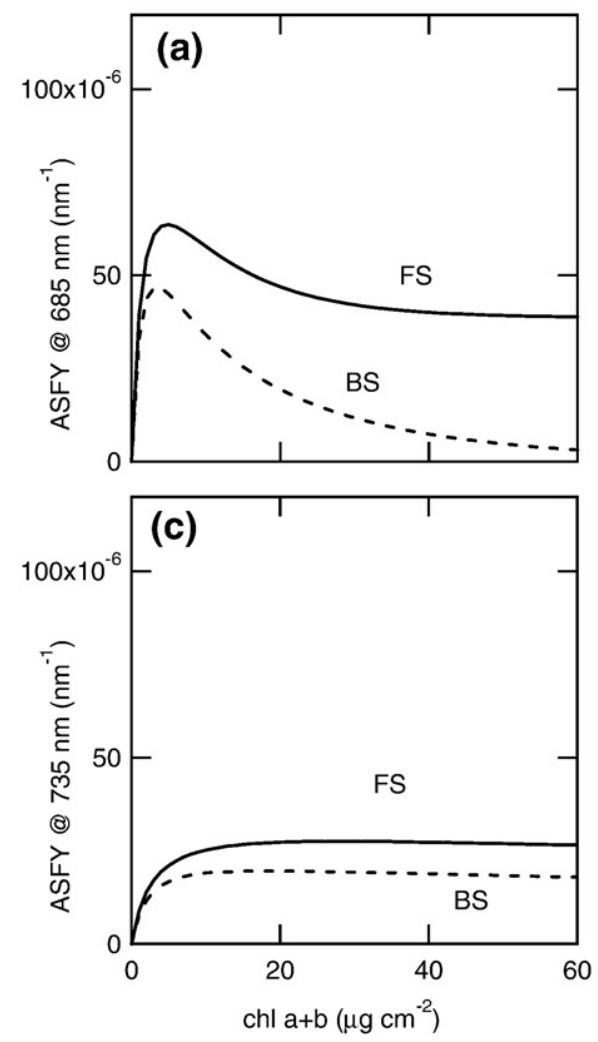

NO DIFFUSION
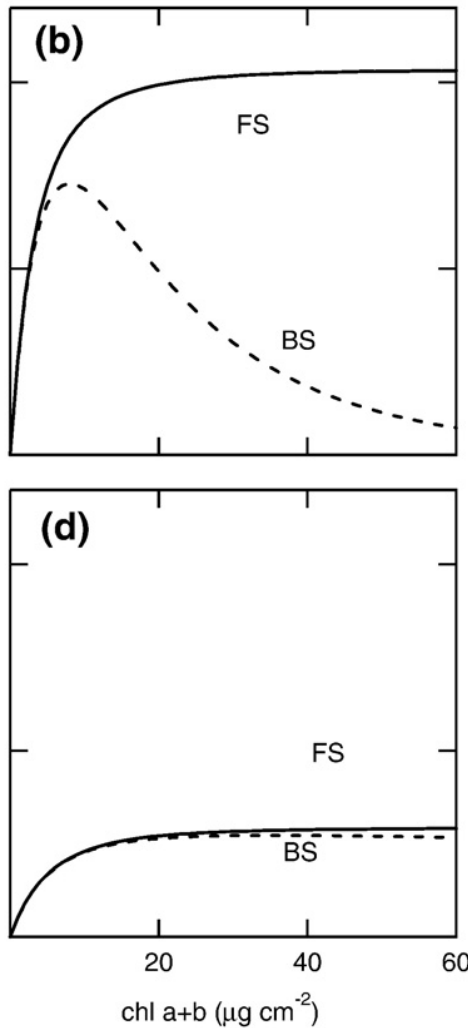

Fig. 10. Fluorescence emission as a function of chlorophyll $a+b$ content $(\mathrm{a}-\mathrm{c}$ ) with diffusion and (b-d) without diffusion. Solid line: front-side fluorescence. Dashed lines: back-side fluorescence. (a-b) $\lambda_{e m}=685 \mathrm{~nm}$ and (c-d) $\lambda_{e m}=735 \mathrm{~nm}$. The other parameters are defined in Table 1.

\subsection{Comparison between FluorMODleaf and the Kubelka-Munk theory}

Among the others alternative radiative transfer models used to simulate light propagation within plant leaves, the KM theory (Kubelka \& Munk, 1931) is the most popular. In this approach, radiation inside a homogeneous medium is modeled by two fluxes traveling in opposite directions and the leaf is characterized by an absorption coefficient $k$ and a scattering coefficient $s$. At any wavelength $\lambda$, the reflectance $R(\lambda)$ and transmittance $T(\lambda)$ can be derived analytically as a function of these two coefficients. An adaptation of the KM theory to fluorescing media has been introduced by Allen (1964), whereas Fukshansky and Kazarinova (1980) and Rosema et al. (1991) extended it to the case of a leaf. Here we use an analytical expression of the first order fluorescence emission, called KMF, derived by integration of the KM propagation equation with Mathematica (Wolfram Research, Inc., Champaign, USA) (see Appendix A). Given a set of input variables for FluorMODleaf, we calculate the leaf reflectance and transmittance at the excitation and emission wavelengths using PROSPECT, and deduce $k$ and $s$ at the same wavelengths by numerical inversion of the KM equations. Then the fluorescence emission computed using KMF is compared to FluorMODleaf outputs for different chlorophyll contents. Fig. 11a and b shows simulations performed at $685 \mathrm{~nm}$ and $735 \mathrm{~nm}$ in the front-side and back-side configurations. In the KMF model, F685 in the front-side configuration does not decrease with $C_{a b}$, which contradicts FluorMODleaf
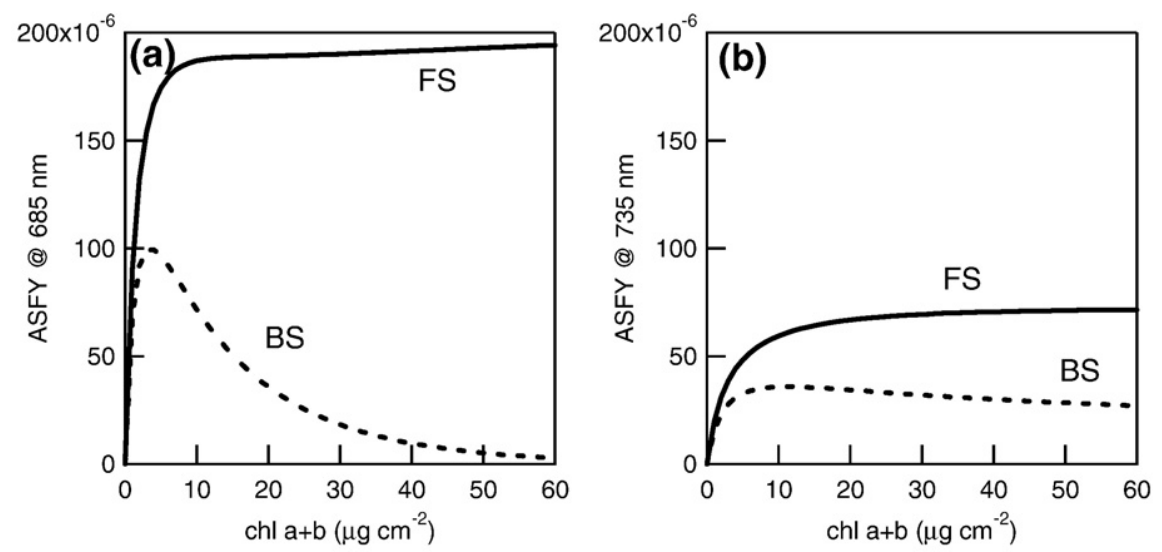

Fig. 11. Fluorescence emission as a function of chlorophyll $a+b$ content in the context of the Kubelka-Munk theory. $\lambda_{e x}=440 \mathrm{~nm}$. The other parameters are defined in Table 1 . (a) $\lambda_{e m}=685 \mathrm{~nm}$. (b) $\lambda_{e m}=735 \mathrm{~nm}$. Solid lines: front-side fluorescence (FS). Dashed lines: back-side fluorescence (BS). 
and data previously reported in the literature (Gitelson et al., 1998; Louis, 2004). The comparison between the two models indicates that the coupling between absorption (or reabsorption) and scattering plays an important role in the fluorescence emission of plant leaves. In FluorMODleaf, scattering and absorption occur in separate spatial regions: scattering takes place at the interfaces between the medium and the air, while absorption is limited to the inner medium. On the contrary, in the KMF theory, absorption and scattering are interlinked at every point inside the medium, which results in a different behavior. The comparison between the two approaches suggests that leaf absorption and scattering occur in distinct regions: in chloroplasts where little scattering occurs (absorption) and at the interface between two media separated by different optical indexes (scattering).

\section{Conclusion}

FluorMODleaf is a new effective chlorophyll $a$ fluorescence emission model that simulates the apparent spectral fluorescence yield (ASFY) of a fresh leaf between $640 \mathrm{~nm}$ and $840 \mathrm{~nm}$, for any excitation light of the visible spectrum. It is based on PROSPECT, a radiative transfer code that has already proven to generate accurate reflectance and transmittance spectra in the solar domain, and the input variables of which - leaf structure parameter and leaf biochemical content - are well identified. Three new variables have been introduced to drive the emission of light: a stoichiometry factor $\sigma_{I I} / \sigma_{I}$ and the two photosystem lifetimes $\tau_{I}$ and $\tau_{I I}$. They have a great influence on fluorescence emission but are difficult to assess because the response of the photosynthetic apparatus to them is species dependent and subject to environmental factors such as air temperature, light intensity, etc. For instance, the distribution of air temperature inside a plant canopy which, to some extent, determines the leaf surface temperature is still difficult to measure or model. FluorMODleaf is certainly the first version of a model that will include in the future plant physiology and environmental conditions such as light level, leaf temperature, etc. This means that an additional effort will be necessary to describe the dependency of these variables with respect to the physiological parameters of the species of interest.

The model outputs have been successfully compared to actual measurements for single-wavelength excitations. They reproduce the variations in fluorescence emission spectra associated with chlorophyll content, particularly those caused by a modification in the reabsorption. This work also highlights the importance of scattering that causes spectral deformations that a cursory analysis can easily pass off as something else. Finally, for sunlight excitation (results not shown) the fluorescence levels predicted by the model are consistent with the values published by Kim et al. (1993).

Last but not least, the next step in this ongoing development will consist in coupling FluorMODleaf with FluorSAIL, the counterpart of the SAIL model for fluorescence (Miller et al., 2005), to simulate light emission by a plant canopy. This unique and powerful simulation tool will improve our understanding of vegetation fluorescence for plant status monitoring.

\section{Acknowledgements}

This work has been funded by the European Space Agency under the contract number 16365/02/NL/FF. We thank J. Breton and A. Krieger for kindly providing us with samples of PSI and PSII preparations. IPGP contribution no. 2509.

\section{Appendix A. Analytical resolution of the Kubelka-Munk equation in the case of a fluorescent medium}

The Kubelka-Munk (KM) theory was developed by Kubelka and Munk (1931) to describe the radiative transfer in a painting medium, later found numerous applications in modeling of the optical properties of thin diffuse medium like paper sheets, ink or plant leaves. In the KM theory, radiation within the medium consists of two diffusive fluxes $I$ and $J$ propagating in opposite directions. In the case where there is no fluorescence, $I$ and $J$ can be easily expressed as a linear combination of exponentials function of depth $z$ inside a horizontal slab. Allen (1964) modified the KM equations to include fluorescence. The flow of total diffuse flux across a horizontal slab of thickness $d z$ at any wavelength $\lambda$ can be written in a differential form as:

$\left\{\begin{array}{l}\frac{d I}{d z}=-(k+s) I+s J+\frac{1}{2} P \\ -\frac{d J}{d z}=s I-(k+s) J+\frac{1}{2} P\end{array}\right.$

where $s$ and $k$ are the linear scattering and absorption coefficients for diffuse light $\left(\mathrm{mm}^{-1}\right), I$ and $J$ are the upward and downward radiant fluxes at depth $z$, and $P$ is the fluorescence emission flux (assumed to be isotropic) at depth $z$. By introducing $P$, we assume that all absorbed photons of $I$ and $J$ in the PAR region contribute to the excitation of the photosystems. $P$ is defined as an integral over the wavelength range 400-700 nm:

$P(z)=\phi \eta \int_{400}^{700} k(I+J) d \lambda$

where $\phi$ is the photon fluorescence efficiency, i.e., the fraction of absorbed upward and downward fluxes that contributes to fluorescence excitation, and $\eta$ is the fluorescence emission spectral distribution function. Fukshansky and Kazarinova (1980), Bonham (1986), and Shakespeare and Shakespeare (2003) analytically solved the extended KM equations by successive approximations. Rosema et al. (1991) and later Zarco-Tejada et al. (2000) preferred a numerical procedure called the "doubling method". Finally Emmel (1998, 2000), Emmel and Hersch (1998) proposed a matrix method. Of course, all these methods should provide the same results. Here we proposed a full analytical solution of the extended KM equation in the case of a weakly fluorescent medium, as is ChlF in leaves. In this case, Eq. (A2) can be reformulated as:

$P(z)=\phi \eta \int_{400}^{700} k\left(I_{0}+J_{0}\right) d \lambda$

where $I_{0}$ and $J_{0}$ are the upward and downward fluxes when fluorescence is neglected. They can be calculated by solving the KM equations of a non fluorescent medium. The general problem can then be reformulated by considering fluorescence emitted at an emission wavelength $\lambda_{2}$ upon excitation with a monochromatic light at a wavelength $\lambda_{1}$. The set of Eq. (A1) is then rewritten as:

$\left\{\begin{aligned} & \frac{d I\left(\lambda_{1}\right)}{d z}=-\left(k_{1}+s_{1}\right) I\left(\lambda_{1}\right)+s_{1} J\left(\lambda_{1}\right) \\ &-\frac{d J\left(\lambda_{1}\right)}{d z}=s_{1} I\left(\lambda_{1}\right)-\left(k_{1}+s_{1}\right) J\left(\lambda_{1}\right) \\ & \frac{d I\left(\lambda_{2}\right)}{d z}=-\left(k_{2}+s_{2}\right) I\left(\lambda_{2}\right)+s_{2} J\left(\lambda_{2}\right)+\frac{1}{2} P\left(\lambda_{1}, \lambda_{2}\right) \\ &-\frac{d J\left(\lambda_{2}\right)}{d z}=s_{2} I\left(\lambda_{2}\right)-\left(k_{2}+s_{2}\right) J\left(\lambda_{2}\right)+\frac{1}{2} P\left(\lambda_{1}, \lambda_{2}\right) .\end{aligned}\right.$

The general solution upon excitation with a polychromatic light can be found by summing solutions obtained with monochromatic light for all wavelengths which make up the polychromatic light. The first two equations of Eq. (A4) are nothing but the KM equations at the excitation wavelength. Their solutions can be expressed analytically in terms of a combination of exponentials depending 
on depth $\mathrm{z}$, and reintroduced in the last two equations of Eq. (A4) considering that:

$P\left(\lambda_{1}, \lambda_{2}\right)=\phi \eta\left(\lambda_{2}\right) k_{1}\left(I\left(\lambda_{1}\right)+J\left(\lambda_{1}\right)\right)$

The last two equations of Eq. (A4) are non homogeneous first order linear differential equations, with a second member which depends exponentially on the variable $z$, and as such, have solutions in terms of a combination of exponentials. We used Mathematica (Wolfram Research, Inc, Champaign, USA) to compute an analytical expression of the solutions of Eq. (A4). Such solution can be entered into a spreadsheet for calculation of fluorescence emission in the frame of the KM theory. The following mathematical formulae are derived from this solution:

$$
\begin{aligned}
& \beta_{1}=s_{1} \sqrt{\left(\frac{k_{1}+s_{1}}{s_{1}}\right)^{2}-1} \\
& \beta_{2}=s_{2} \sqrt{\left(\frac{k_{2}+s_{2}}{s_{2}}\right)^{2}-1} \\
& t_{1}=k_{1}+2 s_{1} \\
& t_{2}=k_{2}+2 s_{2} \\
& u_{1}=k_{1}+s_{1}+\beta_{1} \\
& u_{2}=k_{2}+s_{2}+\beta_{2} \\
& v_{1}=k_{1}+s_{1}-\beta_{1} \\
& v_{2}=k_{2}+s_{2}-\beta_{2} \\
& f_{1}=4 \beta_{1} \beta_{2}\left(t_{2}-t_{1}\right) \\
& f_{2}=\left(t_{1}+\beta_{1}\right)\left(\beta_{1}\left(\beta_{2}+t_{2}\right)-t_{2}\left(\beta_{2}+k_{2}\right)\right) \\
& f_{3}=\left(t_{1}+\beta_{1}\right)\left(\beta_{1}\left(\beta_{2}-t_{2}\right)-t_{2}\left(\beta_{2}-k_{2}\right)\right) \\
& f_{4}=\left(t_{1}-\beta_{1}\right)\left(\beta_{1}\left(\beta_{2}+t_{2}\right)+t_{2}\left(\beta_{2}+k_{2}\right)\right) \\
& f_{5}=\left(t_{1}-\beta_{1}\right)\left(\beta_{1}\left(\beta_{2}-t_{2}\right)+t_{2}\left(\beta_{2}-k_{2}\right)\right) \\
& g_{1}=4 \beta_{1} \beta_{2}\left(\left(\beta_{2}-t_{2}\right)\left(k_{2}+t_{1}\right)+2 \beta_{2} s_{2}\right) \\
& g_{2}=4 \beta_{1} \beta_{2}\left(\left(\beta_{2}+t_{2}\right)\left(k_{2}+t_{1}\right)+2 \beta_{2} s_{2}\right) \\
& g_{3}=2\left(\beta_{1}-t_{1}\right)\left(\beta_{1}-t_{2}\right)\left(k_{2} t_{2}+\beta_{2}^{2}\right) \\
& g_{4}=-2\left(\beta_{1}+t_{1}\right)\left(\beta_{1}+t_{2}\right)\left(k_{2} t_{2}+\beta_{2}^{2}\right) \\
& g_{5}=\left(\beta_{1}+t_{1}\right)\left(\beta_{1}+t_{2}\right)\left(k_{2} t_{2}-\beta_{2}^{2}\right) \\
& g_{6}=-\left(\beta_{1}-t_{1}\right)\left(\beta_{1}-t_{2}\right)\left(k_{2} t_{2}-\beta_{2}^{2}\right) \\
& F_{F S}=\frac{\phi \eta\left(\lambda_{2}\right) k_{1}}{2\left(\beta_{1}-\beta_{2}\right)\left(\beta_{1}+\beta_{2}\right)} \frac{f_{1}+f_{2} e^{\beta_{1}+\beta_{2}}+f_{3} e^{\beta_{1}-\beta_{2}}+f_{4} v^{-\beta_{1}+\beta_{2}}+f_{5} e^{-\beta_{1}-\beta_{1}-\beta_{2}}-v_{1} u_{2} e^{-\beta_{1}+\beta_{2}}-u_{1} v_{2} e^{\beta_{1}-\beta_{2}}+u_{1} u_{2} e^{\beta_{1}+\beta_{2}}}{v_{2}} \\
& F_{B S}=\frac{\phi \eta\left(\lambda_{2}\right) k_{1}}{4 \beta_{2}\left(\beta_{1}-\beta_{2}\right)\left(\beta_{1}+\beta_{2}\right)} \\
& \frac{g_{1} e^{\beta_{1}-\beta_{2}}+g_{2} e^{\beta_{1}}+\beta_{2}+g_{3}+g_{4} e^{2 \beta_{1}}+g_{5}\left(e^{2 \beta_{1}-2 \beta_{2}}+e^{2 \beta_{1}+2 \beta_{2}}\right)+g_{6}\left(e^{2 \beta_{2}}+e^{-2 \beta_{2}}\right)}{-v_{1} v_{2} e^{-\beta_{2}}+v_{1} u_{2} e^{\beta_{2}}+u_{1} v_{2} e^{2 \beta_{1}-\beta_{2}}-u_{1} u_{2} e^{2 \beta_{1}+\beta_{2}}}
\end{aligned}
$$

\section{References}

Agati, G., Fusi, F., Mazzinghi, P., \& Dipaolo, M. L. (1993). A simple approach to the evaluation of the reabsorption of chlorophyll fluorescence spectra in intact leaves. Journal of Photochemistry and Photobiology B, 17, 163-171.

Agati, G., Cerovic, Z., \& Moya, I. (2000). The effect of decreasing temperature up to chilling values on the in vivo F685/F735 chlorophyll fluorescence ratio in Phaseolus vulgaris and Pisum sativum: The role of the photosystem I contribution to the $735 \mathrm{~nm}$ fluorescence band. Photochemistry and Photobiology, 72, 75-84.

Allen, E. (1964). Fluorescent white dyes: Calculation of fluorescence from reflectivity values. Journal of the Optical Society of America, 54, 506-515.

Allen, W. A., Gausman, H. W., Richardson, A. J., \& Wiegand, C. L. (1970). Mean effective optical constants of thirteen kinds of plant leaves. Applied Optics, 9, 2573-2577.

Anderson, J. M., \& Melis, A. (1983). Localization of different photosystems in separate regions of chloroplast membranes. Proceedings of the National Academy of Sciences of the United States of America-Biological Sciences, 80, 745-749.
Bassi, R., Silvestri, M., Dainese, P., Moya, I., \& Giacometti, G. M. (1991). Effects of a nonionic detergent on the spectral properties and aggregation state of the lightharvesting chlorophyll-a/b protein complex (LHCII). Journal of Photochemistry and Photobiology B-Biology, 9, 335-354.

Benford, F. (1923). Reflection and transmission by parallel plates. Journal of the Optical Society of America, 7, 1017-1025.

Berthold, D. A., Babcock, G. T., \& Yocum, C. F. (1981). A highly resolved, oxygen-evolving photosystem II preparation from spinach thylakoid membranes. FEBS Letter, 134, $231-234$.

Bonham, J. S. (1986). Fluorescence and Kubelka-Munk theory. Color Research and Application, 11, 223-230.

Borisov, A. Y., \& Il'ina, M. D. (1973). The fluorescence lifetime and energy migration mechanism in photosystem I of plants. Biochimica et Biophysica Acta, 305, 364-371.

Brody, S., \& Rabinowitch, E. (1957). Excitation lifetime of photosynthetic pigments in vitro and in vivo. Science, 125, 555-555.

Buschmann, C. (2007). Variability and application of the chlorophyll fluorescence emission ratio red/far-red of leaves. Photosynthesis Research, 92, 261-271.

Byrdin, M., Rimke, I., Schlodder, E., Stehlik, D., \& Roelofs, T. A. (2000). Decay kinetics and quantum yields of fluorescence in photosystem I from Synechococcus elongatus with P700 in the reduced and oxidized state: Are the kinetics of excited state decay trap-limited or transfer-limited? Biophysical Journal, 79, 992-1007.

Cerovic, Z. G., Samson, G., Morales, F., Tremblay, N., \& Moya, I. (1999). Ultravioletinduced fluorescence for plant monitoring: Present state and prospects. Agronomie $19,543-578$.

Chow, W. S., Melis, A., \& Anderson, J. M. (1990). Adjustments of photosystem stoichiometry in chloroplasts improve the quantum efficiency of photosynthesis. Proceedings of the National Academy of Sciences of the United States of America, 87, 7502-7506.

Cordon, G. B., \& Lagorio, M. G. (2006). Re-absorption of chlorophyll fluorescence in leaves revisited. A comparison of correction models. Photochemical \& Photobiological Sciences, 5, 735-740.

Cordon, G. B., \& Lagorio, M. G. (2007). Absorption and scattering coefficients: A biophysical-chemistry experiment using reflectance spectroscopy. Journal of Chemical Education, 84, 1167-1170.

Cordon, G. B., \& Lagorio, M. G. (2007). Optical properties of the adaxial and abaxial faces of leaves. Chlorophyll fluorescence, absorption and scattering coefficients. Photochemical \& Photobiological Sciences, 6, 873-882.

Crilly, R. J., Cheong, W., Wilson, B., \& Spears, J. R. (1997). Forward-adjoint fluorescence model: Monte Carlo integration and experimental validation. Applied Optics, 36, 6513-6519.

Croce, R., Zucchelli, G., Garlaschi, F. M., Bassi, R., \& Jennings, R. C. (1996). Excited state equilibration in the photosystem I-light-harvesting I complex: P700 is almost isoenergetic with its antenna. Biochemistry, 35, 8572-8579.

Danielsson, R., Albertsson, P. A., Mamedov, F. \& Styring, S. (2004). Quantification of photosystem I and II in different parts of the thylakoid membrane from spinach. Biochimica et Biophysica Acta - Bioenergetics, 1608, 53-61.

Davidson, M., Berger, M., Moya, I., Moreno, J., Laurila, T., Stoll, M. P., et al. (2003) Mapping photosynthesis from space - A new vegetation-fluorescence technique. ESA Bulletin, 116, 34-37.

Duysens, L. N. M. (1952). Transfer of excitation energy in photosynthesis. PhD Thesis Utrecht University (Utrecht, The Netherlands).

Emerson, R., Chalmers, R., \& Cederstrand, C. (1957). Some factors influencing the longwave limit of photosynthesis. Proceedings of the National Academy of Sciences USA, 43, $133-143$.

Emmel, P. (1998). Modèles de prédiction couleur appliqués à l'impression jet d'encre PhD Thesis, Ecole Polytechnique Fédérale de Lausanne, Lausanne (Suisse), 172 pp.

Emmel, P., \& Hersch, R. D. (1998). Spectral colour prediction model for a transparen fluorescent ink on paper. Proceedings of the 6th Color Imaging Conference: Color Science, Systems and Applications, 17-20 November 1998, Scottsdale (AZ, USA) (pp. 116-122).

Emmel, P. (2000). Nouvelle formulation du modèle de Kubelka et Munk avec applications aux encres fluorescentes. Actes de l'Ecole de Printemps 2000 - Le pays d'Apt en couleurs, Apt en Roussillon (France)

Feret, J B., François, C., Asner, G. P. Gitelson, A. A, Martin, R. E, Bidel, L. P. R., et al. (2008) PROSPECT-4 and 5: Advances in the leaf optical properties model separating photosynthetic pigments. Remote Sensing of Environment, 112, 3030-3043.

Flexas, J., Briantais, J. M., Cerovic, Z., Medrano, H., \& Moya, I. (2000). Steady-state and maximum chlorophyll fluorescence responses to water stress in grapevine leaves: A new remote sensing system. Remote Sensing of Environment, 73, 283-297.

Franck, F., Juneau, P., \& Popovic, R. (2002). Resolution of the photosystem I and photosystem II contributions to chlorophyll fluorescence of intact leaves at room temperature. Biochimica et Biophysica Acta, 1556, 239-246.

Fukshansky, L., \& Kazarinova, N. (1980). Extension of the Kubelka-Munk theory of light propagation in intensely scattering materials to fluorescent media. Journal of the Optical Society of America, 70, 1101-1111.

Gardner, C. M., Jacques, S. L., \& Welch, A. J. (1996). Fluorescence spectroscopy of tissue: Recovery of intrinsic fluorescence from measured fluorescence. Applied Optics, 35 , $1780-1792$.

Georgakoudi, I. (2006). The color of cancer. Journal of Luminescence, 119, 75-83.

Gitelson, A. A., Buschmann, C., \& Lichtenthaler, H. K. (1998). Leaf chlorophyl fluorescence corrected for re-absorption by means of absorption and reflectance measurements. Journal of Plant Physiology, 152, 283-296.

Gitelson, A. A., Merzlyak, M. N., \& Chivkunova, O. B. (2001). Optical properties and nondestructive estimation of anthocyanin content in plant leaves. Photochemistry and Photobiology, 74, 38-45.

Goulas, Y. (1992). Télédétection de la fluorescence des couverts végétaux: temps de vie de la fluorescence chlorophyllienne et fluorescence bleue. PhD Thesis, Université de Paris-Sud (Orsay, France), 198 pp. 
Grace, J., Nichol, C., Disney, M., Lewis, P., Quaife, T., \& Bowyer, P. (2007). Can we measure terrestrial photosynthesis from space directly, using spectral reflectance and fluorescence? Global Change Biology, 13, 1484-1497.

Guanter, L., Alonso, L., Gómez-Chova, L., Amorós-López, J., Vila, J., \& Moreno, J. (2007). Estimation of solar-induced vegetation fluorescence from space measurements. Geophysical Research Letters, 34, L08401.

Gupta, S., Raja, V., \& Pradhan, A. (2006). Simultaneous extraction of optical transport parameters and intrinsic fluorescence of tissue mimicking model media using a spatially resolved fluorescence technique. Applied Optics, 45, 7529-7537.

Hodges, M., \& Moya, I. (1988). Time-resolved chlorophyll fluorescence studies on pigment-protein complexes from photosynthetic membranes. Biochimica and Biophysica Acta, 935, 41-52.

Jacquemoud, S., \& Baret, F. (1990). PROSPECT: A model of leaf optical properties spectra. Remote Sensing of Environment, 34, 75-91.

Kim, M. S., Chappelle, E. W., Corp, L., \& McMurtrey, J. E., III (1993). The contribution of chlorophyll fluorescence to the reflectance spectra of green vegetation. Proceedings of the International Geoscience and Remote Sensing Symposium (IGARSS '93), 3, 1321-1224.

Krause, G. H., \& Weis, E. (1991). Chlorophyll fluorescence and photosynthesis: The basics. Annual Review of Plant Physiology and Plant Molecular Biology, 42, 313-349.

Kubelka, P., \& Munk, F. (1931). Ein Beitrag zur Optik der Farbanstriche. Zeitschrift für Technische Physik, 12, 593-601.

Laasch, H. (1987). Non-photochemical quenching of chlorophyll a fluorescence in isolated chloroplasts under conditions of stressed photosynthesis. Planta, 171, 220-226.

Leong, T. Y., \& Anderson, J. M. (1984). Adaptation of the thylakoid membranes of pea chloroplasts to light intensities. I. Study on the distribution of chlorophyll-protein complexes. Photosynthesis Research, 5, 105-115.

Lokstein, H., Tian, L., Polle, J. E. W., \& DellaPenna, D. (2002). Xanthophyll biosynthetic mutants of Arabidopsis thaliana: Altered nonphotochemical quenching of chlorophyll fluorescence is due to changes in photosystem II antenna size and stability. Biochimica et Biophysica Acta - Bioenergetics, 1553, 309-319.

Louis, J. (2004). Télédétection et modélisation des signaux de fluorescence et de réflectance (PRI) des couverts végétaux. PhD Thesis, Université Paris Diderot Paris 7, Paris (France), $201 \mathrm{pp}$.

Louis, J., Cerovic, Z. G., \& Moya, I. (2006). Quantitative study of fluorescence excitation and emission spectra of bean leaves. Journal of Photochemistry and Photobiology B, $85,65-71$.

Maier, S. W. (2000). Modeling the radiative transfer in leaves in the $300 \mathrm{~nm}$ to $2.5 \mu \mathrm{m}$ wavelength region taking into consideration chlorophyll fluorescence - The leaf model SLOPE. PhD Thesis, Technische Universität München, Oberpfaffenhofen (Germany), 110 pp.

Melis, A., \& Brown, J. S. (1980). Stoichiometry of system I and system II reaction centers and of plastoquinone in different photosynthetic membranes. Proceedings of the National Academy of Sciences of the United States of America-Biological Sciences, 77, $4712-4716$

Melis, A. (1989). Spectroscopic methods in photosynthesis: Photosystem stoichiometry and chlorophyll antenna size. Philosophical Transactions of the Royal Society of London Series B-Biological Sciences, 323, 397-409.

Miller, J., Berger, M., Goulas, Y., Jacquemoud, S., Louis, J., Mohammed, G., et al. (2005). Development of a vegetation fluorescence canopy model ESTEC Contract No. 16365/02/NL/FF, (http://www.ias.csic.es/fluormod/documents/ FluorMOD_Final_Report_April_13_2005.pdf), 138 pp.

Monteith, J., \& Unsworth, M. (2007). Principles of environmental physics, Third Edition: Academic Press 440 pp.

Moya, I. (1974). Durée de vie et rendement de fluorescence de la chlorophylle in vivo. Leur relation dans différents modèles d'unités photosynthétiques. Biochimica et Biophysica Acta, 368, 214-227.
Moya, I., Hodges, M., \& Barbet, J. C. (1986). Modification of room-temperature picosecond chlorophyll fluorescence kinetics in green algae by photosystem II trap closure. FEBS letters, 198, 256-262.

Moya, I., Guyot, G., \& Goulas, Y. (1992). Remotely sensed blue and red fluorescence emission for monitoring vegetation. ISPRS Journal of Photogrammetry and Remote Sensing, 47, 205-231.

Moya, I., Silvestri, M., Vallon, O., Cinque, G., \& Bassi, R. (2001). Time-resolved fluorescence analysis of the photosystem II antenna proteins in detergent micelles and liposomes. Biochemistry, 40, 12552-12561.

Moya, I., \& Cerovic, Z. G. (2004). Remote sensing of chlorophyll fluorescence: Instrumentation and analysis. In G. C. Papageorgiou X Govindjee (Eds.), Chlorophyll a fluorescence: A signature of photosynthesis (pp. 429-445). Springer.

Mullet, J. E., Burke, J. J., \& Arntzen, C. J. (1980). Chlorophyll proteins of photosystem I. Plant Physiology, 65, 814-822.

Ounis, A., Cerovic, Z. G., Briantais, J. M., \& Moya, I. (2001). Dual-excitation FLIDAR for the estimation of epidermal UV absorption in leaves and canopies. Remote Sensing of Environment, 76, 33-48.

Panou-Diamandi, O., Uzunoglu, N. K., Zacharakis, G., Filippidis, G., Papazoglou, T., \& Koutsouris, D. (1998). A one layer tissue fluorescence model based on electromagnetic theory. Journal of Electromagnetic Waves \& Applications, 12, 1101-1121.

Pedrós, R., Moya, I., Goulas, Y., \& Jacquemoud, S. (2008). Chlorophyll fluorescence emission spectrum inside a leaf. Photochemical \& Photobiological Sciences, 7, 498-502.

Ramos, M. E., \& Lagorio, M. G. (2004). True fluorescence spectra of leaves. Photochemical E' Photobiological Sciences, 3, 1063-1066.

Richards-Kortum, R., Rava, R. P., Fitzmaurice, M., Tong, L. L., Ratliff, N. B., Kramer, J. R., et al. (1989). A one-layer model of laser-induced fluorescence for diagnosis of disease in human tissue: Applications to atherosclerosis. IEEE Transactions on Bio-Medical Engineering, 36, 1222-1232.

Rosema, A., Verhoef, W., Schroote, J., \& Snel, J. F. H. (1991). Simulating fluorescence light-canopy interaction in support of laser-induced fluorescence measurements. Remote Sensing of Environment, 37, 117-130.

Schmuck, G., Verdebout, J., Koechler, C., Moya, I., \& Goulas, Y. (1991). Laser-induced time resolved fluorescence of vegetation. IEEE Transactions on Geoscience and Remote Sensing, 29, 674-678.

Schmuck, G., \& Moya, I. (1994). Time-resolved chlorophyll fluorescence spectra of intact leaves. Remote Sensing of Environment, 47, 72-76.

Shakespeare, T., \& Shakespeare, J. (2003). A fluorescent extension to the Kubelka-Munk model. Color Research and Application, 28, 4-14.

Sušila, P., \& Nauš, J. (2007). A Monte Carlo study of the chlorophyll fluorescence emission and its effect on the leaf spectral reflectance and transmittance under various conditions. Photochemical \& Photobiological Sciences, 6, 894-902.

Tumerman, L. A., \& Sorokin, E. M. (1967). The photosynthetic unit: A "physical" or "statistical" model? Molecular Biology (USSR), 1, 628-638.

Virgin, H. I. (1954). The distortion of fluorescence spectra in leaves by light scattering and its reduction by infiltration. Physiologia Plantarum, 7, 560-570.

Vogelmann, T. C. (1989). Penetration of light into plants. Photochemistry and Photobiology, 50, 895-902.

Welch, A. J., Gardner, C., Richards-Kortum, R., Chan, E., Criswell, G., Pfefer, J., et al. (1997). Propagation of fluorescent light. Lasers in Surgery and Medicine, 21, 166-178.

Wu, J., Feld, M. S., \& Rava, R. P. (1993). An analytical model for extracting intrinsic fluorescence in a turbid media. Applied Optics, 32, 3585-3595.

Yamazaki, J., Kamimura, Y., Okada, M., \& Sugimura, Y. (1999). Changes in photosynthetic characteristics and photosystem stoichiometries in the lower leaves in rice seedlings. Plant Science, 148, 155-163.

Zarco-Tejada, P. J., Miller, J. R., Mohammed, G. H., \& Noland, T. L. (2000). Chlorophyll fluorescence effects on vegetation apparent reflectance: I. Leaf-level measurements and model simulation. Remote Sensing of Environment, 74, 582-595. 